\title{
New black-string solutions for an anti-de Sitter brane in scalar-tensor gravity
}

\author{
Theodoros Nakas, ${ }^{1, *}$ Nikolaos Pappas, ${ }^{2, \dagger}$ and Panagiota Kanti ${ }^{1,+}$ \\ ${ }^{1}$ Division of Theoretical Physics, Department of Physics, University of Ioannina, \\ Ioannina GR-45110, Greece \\ ${ }^{2}$ Nuclear and Particle Physics Section, Physics Department, \\ National and Kapodistrian University of Athens, Athens GR-15771, Greece
}

(Received 5 April 2019; published 25 June 2019)

\begin{abstract}
We consider a five-dimensional theory with a scalar field nonminimally coupled to gravity, and we look for novel black-string solutions in the bulk. By appropriately choosing the nonminimal coupling function of the scalar field, we analytically solve the gravitational and scalar-field equations in the bulk to produce black-string solutions that describe a Schwarzschild-anti-de Sitter space-time on the brane. We produce two complete such solutions that are both characterized by a regular scalar field, a localized-close-to-ourbrane energy-momentum tensor and a negative-definite, nontrivial bulk potential that may support by itself the warping of the space-time even in the absence of the traditional, negative, bulk cosmological constant. Despite the infinitely long string singularity in the bulk, the four-dimensional effective theory on the brane is robust, with the effective gravity scale being related to the fundamental one and the warping scale. It is worth noting that if we set the mass of the black hole on the brane equal to 0 , the black string disappears, leaving behind a regular brane-world model with only a true singularity at the boundary of the fifth dimension.
\end{abstract}

DOI: 10.1103/PhysRevD.99.124040

\section{INTRODUCTION}

The general theory of relativity [1-3] is a mathematically beautiful, tensorial theory of gravity that predicts a variety of fascinating gravitational objects such as black holes, wormholes or compact stars. It was formulated in the $(3+1)$ space-time dimensions of our world but it has since been extended to an arbitrary number of extra dimensions [4-12]. Although the mathematical extension of the theory to a higher number of dimensions was straightforward, the implications on the physical properties of the derived solutions have been quite dramatic. Black-hole solutions are a characteristic example of such a transition: whereas in four dimensions these solutions have been uniquely determined and classified (see, for example, $[13,14]$ ), in higher number of dimensions, they are merely one species of the family of the so-called black objects, which includes black holes, black strings, black branes, black rings, or black

\footnotetext{
thnakas@cc.uoi.gr †npappas@cc.uoi.gr "pkanti@cc.uoi.gr
}

Published by the American Physical Society under the terms of the Creative Commons Attribution 4.0 International license. Further distribution of this work must maintain attribution to the author(s) and the published article's title, journal citation, and DOI. Funded by SCOAP . saturns [15]. The study of the properties of all these objects is still a topic of intensive research activity and the formulation of uniqueness theorems or their complete classification is still lacking.

In fact, the determination of regular black-hole solutions in the context of the so-called brane-world models $[11,12]$ has been a challenging task that, in part, remains still unfulfilled. These models present the additional complication of the presence of the self-energy of the brane, which is one of the fundamental features of the theory as it gives rise to the desired warping of space-time. This feature has so far prevented the determination of an analytical, nonapproximate solution describing a regular, localized-close-to-our-brane black hole. Although a large number of different theoretical approaches and techniques have been used (see Refs. [16-49] for an impartial list of works), only numerical solutions describing, in principle, regular black holes have been found [50-56]. It is worth noting that, in the context of theories where the brane selfenergy may be ignored [8-10], analytical forms of higherdimensional black holes were derived long ago $[57,58]$ and the study of their properties, either classical or quantum, has led to a separate direction of research activity.

Whereas analytical solutions describing higherdimensional black holes in a warped space-time are still elusive, the other members of the family of black objects 
have been easier to find [15]. For example, the first black-string solution in the context of the models [11,12] was derived in [16]: although from the point of view of a four-dimensional observer that solution described a black hole on the brane, the complete bulk solution had a stringlike singularity extending all the way from the brane to the boundaries of the fifth dimension. This nonphysical gravitational solution was, in addition, plagued by an intrinsic instability that segmented the infinitely extended black string to a sequence of black cigars $[59,60]$. Since then, a variety of higher-dimensional black strings have been derived in the literature-see, for example Refs. [61-76].

The easiness with which classical black-string solutions arise in the context of a brane-world model, as opposed to the difficulty that we face when we look for black-hole solutions, has fueled a great controversy in the literature over the years [77-85], which in fact remains undecided in the absence of an analytical solution describing a regular black hole. Let us consider, for example, a brane-world model that contains a bulk scalar field with an arbitrary potential and a nonminimal coupling to gravity. Braneworld solutions with a Minkowski space-time on our brane ${ }^{1}$ were studied in the context of this theory in [88-91]. In $[34,41]$, the same theory was exhaustively studied for the determination of an analytical solution describing a regular black hole localized close to our brane; apart from the scalar degree of freedom (d.o.f.) that was supplemented by an arbitrary bulk potential, a generalized ansatz for the higher-dimensional line element was employed that allowed for a diverse, and even dynamical, black-hole solution on the brane. Yet, no viable complete bulk solution was found.

In contrast, the analyses of [34,41] have shown that a scalar-tensor theory of gravity in a five-dimensional warped space-time may allow quite easily for new black-string solutions to emerge. Indeed, in [74], we focused on such a theory and looked for analytical bulk solutions of the complete set of gravitational and scalar-field equations. The solution for the five-dimensional line element described indeed a black string reducing to a Schwarzschild-(anti-) de Sitter (AdS) space-time on the brane. We chose to study the case of a positive cosmological constant on the brane, which remarkably resulted in the condition that the nonminimal coupling function of the scalar field to gravity had to be negative over a particular regime in the bulk. Despite the antigravitating force that condition led to, some of the solutions found had a robust four-dimensional effective theory on the brane.

In this work, we focus on the case of a negative cosmological constant on the brane, which as we demonstrate

\footnotetext{
${ }^{1}$ Brane-world models based on a five-dimensional bulk scalar field leading to a cosmologically evolving brane were also studied in $[86,87]$.
}

removes the condition of the negative sign of the nonminimal coupling function in the bulk. With the gravity thus having everywhere the correct sign, we look again for analytical solutions describing novel black strings. We explicitly solve the coupled system of gravitational and scalar-field equations to determine both the bulk gravitational background and the scalar-field configuration. Demanding the regularity of the scalar field everywhere in the bulk, we reduce the general form of the coupling function to two particular choices. Both choices lead to analytical black-string solutions that, apart from the infinitely long string singularity, are free of any additional bulk singularities associated either with the scalar field or with the five-dimensional line element. The solutions that we found exhibit also a number of attractive features: the energy-momentum tensor of the theory is everywhere regular and localized close to our brane, leading to a five-dimensional Minkowski space-time at large distances away from it. Also, the warping of the fifth dimension may be supported exclusively by the negative-definite, nontrivial bulk potential of the scalar field, a result that makes redundant the presence of the negative bulk cosmological constant. Finally, the five-dimensional theory leads to a robust four-dimensional effective theory on the brane with the effective gravity scale being related to the fundamental one by a relation almost identical to the one appearing in $[11,12]$. It is worth noting that if we set the mass of the black hole on the brane equal to 0 , the black string disappears leaving behind a regular brane-world model with only a true singularity at the boundary of the fifth dimension.

Our paper has the following outline: in Sec. II, we present our theory, the field equations, and set a number of physical constraints on the scalar field and its coupling function. In Sec. III, we study in detail the case of an exponential coupling function and determine the complete bulk solution, its physical properties, the junction conditions, as well as the effective theory on the brane. We repeat the analysis for another interesting case, that of a double exponential coupling function, and discuss its properties in Sec. IV. We finally present our conclusions in Sec. V.

\section{THE THEORETICAL FRAMEWORK}

In this work, we consider a five-dimensional gravitational theory that contains the five-dimensional scalar curvature $R$, a bulk cosmological constant $\Lambda_{5}$, and a five-dimensional scalar field $\Phi$ with a self-interacting potential $V_{B}(\Phi)$. The scalar field also possesses a nonminimal coupling to $R$ via a general coupling function $f(\Phi)$ : this function is initially left arbitrary and is given indicative specific forms when particular physical criteria are set towards the end of this section. Thus, the action functional of the class of theories we consider in this work is given by the expression 


$$
\begin{aligned}
S_{B}= & \int d^{4} x \int d y \sqrt{-g^{(5)}} \\
& \times\left[\frac{f(\Phi)}{2 \kappa_{5}^{2}} R-\Lambda_{5}-\frac{1}{2} \partial_{L} \Phi \partial^{L} \Phi-V_{B}(\Phi)\right] .
\end{aligned}
$$

In the above, $g_{M N}^{(5)}$ is the metric tensor of the fivedimensional space-time, and $\kappa_{5}^{2}=8 \pi G_{5}$ incorporates the five-dimensional gravitational constant $G_{5}$. Our fourdimensional world is represented by a 3-brane located at $y=0$ along the fifth spatial dimension. Thus, in order to complete the model, the following brane action should be added to the bulk one, presented above:

$$
\begin{aligned}
S_{b r} & =\int d^{4} x \sqrt{-g^{(b r)}}\left(\mathcal{L}_{b r}-\sigma\right) \\
& =-\int d^{4} x \int d y \sqrt{-g^{(b r)}}\left[V_{b}(\Phi)+\sigma\right] \delta(y) .
\end{aligned}
$$

For simplicity, the quantity $\mathcal{L}_{b r}$, which is related to the matter/field content of the brane, contains only an interaction term $V_{b}(\Phi)$ of the bulk scalar field with the brane. In addition, $\sigma$ is the constant brane self-energy, and $g_{\mu \nu}^{(b r)}=$ $g_{\mu \nu}^{(5)}\left(x^{\lambda}, y=0\right)$ is the induced-on-the-brane metric tensor. Throughout this work, we denote five-dimensional indices with capital latin letters $M, N, L, \ldots$ and four-dimensional indices with lowercase greek letters $\mu, \nu, \lambda, \ldots$ as usual.

If we vary the complete action $S=S_{B}+S_{b r}$ with respect to the scalar field, we obtain its equation of motion

$$
\begin{aligned}
& -\frac{1}{\sqrt{-g^{(5)}}} \partial_{M}\left(\sqrt{-g^{(5)}} g^{M N} \partial_{N} \Phi\right) \\
& =\frac{\partial_{\Phi} f}{2 \kappa_{5}^{2}} R-\partial_{\Phi} V_{B}-\frac{\sqrt{-g^{(b r)}}}{\sqrt{-g^{(5)}}} \partial_{\Phi} V_{b} \delta(y)
\end{aligned}
$$

The above must be supplemented by the gravitational field equations, which follow from the variation of the action with respect to the metric-tensor components $g_{M N}^{(5)}$, and have the form

$$
\begin{aligned}
f(\Phi) G_{M N} \sqrt{-g^{(5)}}= & \kappa_{5}^{2}\left[\left(T_{M N}^{(\Phi)}-g_{M N} \Lambda_{5}\right) \sqrt{-g^{(5)}}\right. \\
& \left.-\left[V_{b}(\Phi)+\sigma\right] g_{\mu \nu}^{(b r)} \delta_{M}^{\mu} \delta_{N}^{\nu} \delta(y) \sqrt{-g^{(b r)}}\right],
\end{aligned}
$$

where

$$
\begin{aligned}
T_{M N}^{(\Phi)}= & \partial_{M} \Phi \partial_{N} \Phi+g_{M N}\left[-\frac{\partial_{L} \Phi \partial^{L} \Phi}{2}-V_{B}(\Phi)\right] \\
& +\frac{1}{\kappa_{5}^{2}}\left[\nabla_{M} \nabla_{N} f(\Phi)-g_{M N} \square f(\Phi)\right] .
\end{aligned}
$$

In order to derive the explicit form of the aforementioned field equations (3) and (4), we need to specify the form of the five-dimensional gravitational background. We assume that this has the form of a warped space-time with its fourdimensional part having the form of a generalized Vaidya line element. Thus, we consider the expression

$$
\begin{aligned}
d s^{2}= & e^{2 A(y)}\left\{-\left[1-\frac{2 m(r)}{r}\right] d v^{2}+2 d v d r\right. \\
& \left.+r^{2}\left(d \theta^{2}+\sin ^{2} \theta d \varphi^{2}\right)\right\}+d y^{2} .
\end{aligned}
$$

In the above, the function $e^{2 A(y)}$ stands for the warp factor along the fifth dimension, and $m(r)$ is a generalized mass function. A first advantage of the above metric form is that, upon setting the coordinate $y$ along the extra dimension to 0 , the part inside the curly brackets reduces to the line element on the brane. Then, if the function $m(r)$ is assumed to be a constant $M$, the four-dimensional line element is merely the Vaidya transformation of the Schwarzschild solution, where $M$ is the black-hole mass. Despite the fact that the line element on the brane admits such an attractive interpretation, the complete five-dimensional line element, in the context of the purely gravitational Randall-Sundrum model $[11,12]$, was shown to describe a black string [16] plagued also by instabilities $[59,60]$.

Since then, and despite all efforts, no analytical, closed form of a regular, localized-on-the-brane black-hole solution has been found. During that quest, line element (6) was used in a number of works $[24,34,41]$ as it exhibited a second advantage: since it contained no horizon in its four-dimensional part, it did not lead to additional bulk singularities [20,24]. A generalized Vaidya form, where $m$ is not a constant anymore but a function of the coordinates, was employed in an effort to increase the flexibility of the model and allow for brane black-hole solutions to deviate from the overly simple Schwarzschild one. In fact, line element (6) with $m=m(v, r, y)$ was used in [41] in the context of the scalar-tensor theory (1) but, despite its flexibility, which was further increased by the scalar d.o.f., led to no robust black-hole solutions.

As in our previous work [74], here, we also turn our attention to the derivation of novel black-string solutions that the theory (1) seems to possess in abundance. Indeed, in [74] we demonstrated that the theory allows for a variety of such solutions that may be constructed analytically. Assuming that the cosmological constant on the brane is nonvanishing and positive, novel black-string solutions were derived and characterized by interesting properties such as the existence of an antigravitating regime in the bulk. Here, we continue our pursuit for novel black strings in the context of the theory (1) but allow the brane cosmological constant to be negative. We again make the choice $m=m(r)$ for the mass function, and demonstrate by direct integration that a class of black-string 
solutions arises that, on the brane, has the interpretation of a Schwarzschild-anti-de Sitter black hole.

Therefore, employing line element (6) and the relation $\sqrt{-g^{(5)}}=\sqrt{-g^{(4)}}$ that holds in this case, the scalar-field equation of motion (3) in the bulk [i.e., we ignore for now the brane $\delta(y)$-term] takes the explicit form

$$
\begin{aligned}
\Phi^{\prime \prime}+4 A^{\prime} \Phi^{\prime}= & \partial_{\Phi} f\left(10 A^{\prime 2}+4 A^{\prime \prime}-e^{-2 A} \frac{2 \partial_{r} m+r \partial_{r}^{2} m}{r^{2}}\right) \\
& +\partial_{\Phi} V_{B} .
\end{aligned}
$$

In the above, a prime (I) denotes the derivative with respect to the $y$ coordinate. We have also assumed that the bulk scalar field depends only on the coordinate along the fifth dimension, i.e., $\Phi=\Phi(y)$. For the explicit form of the gravitational equations, we need the nonvanishing components of the Einstein $G^{M}{ }_{N}$ and energy-momentum $T^{(\Phi) M}{ }_{N}$ tensors. In mixed form, these are

$$
\begin{aligned}
& G_{0}^{0}=G^{1}{ }_{1}=6 A^{\prime 2}+3 A^{\prime \prime}-\frac{2 e^{-2 A} \partial_{r} m}{r^{2}}, \\
& G_{2}^{2}=G^{3}{ }_{3}=6 A^{\prime 2}+3 A^{\prime \prime}-\frac{e^{-2 A} \partial_{r}^{2} m}{r}, \\
& G^{4}{ }_{4}=6 A^{\prime 2}-\frac{e^{-2 A}\left(2 \partial_{r} m+r \partial_{r}^{2} m\right)}{r^{2}},
\end{aligned}
$$

and

$$
\begin{aligned}
T_{0}^{(\Phi) 0} & =T^{(\Phi) 1}{ }_{1}=T^{(\Phi) 2}{ }_{2}=T^{(\Phi) 3}{ }_{3} \\
& =A^{\prime} \Phi^{\prime} \partial_{\Phi} f+\mathcal{L}_{\Phi}-\square f, \\
T^{(\Phi) 4}{ }_{4} & =\left(1+\partial_{\Phi}^{2} f\right) \Phi^{\prime 2}+\Phi^{\prime \prime} \partial_{\Phi} f+\mathcal{L}_{\Phi}-\square f,
\end{aligned}
$$

respectively. Above, we have defined the quantities

$$
\mathcal{L}_{\Phi}=-\frac{1}{2} \partial_{L} \Phi \partial^{L} \Phi-V_{B}(\Phi)=-\frac{1}{2} \Phi^{\prime 2}-V_{B}(\Phi)
$$

and

$$
\square f=4 A^{\prime} \Phi^{\prime} \partial_{\Phi} f+\Phi^{\prime 2} \partial_{\Phi}^{2} f+\Phi^{\prime \prime} \partial_{\Phi} f .
$$

By substituting the aforementioned components of the Einstein and scalar energy-momentum tensors in Eq. (4), and ignoring again the brane boundary terms, the gravitational field equations in the bulk may be derived. We thus obtain three equations that, upon some simple manipulation [74], assume the following form:

$$
r \partial_{r}^{2} m-2 \partial_{r} m=0,
$$

$f\left(3 A^{\prime \prime}+e^{-2 A} \frac{\partial_{r}^{2} m}{r}\right)=\partial_{\Phi} f\left(A^{\prime} \Phi^{\prime}-\Phi^{\prime \prime}\right)-\left(1+\partial_{\Phi}^{2} f\right) \Phi^{\prime 2}$, $f\left(6 A^{\prime 2}+3 A^{\prime \prime}-\frac{2 e^{-2 A} \partial_{r} m}{r^{2}}\right)=A^{\prime} \Phi^{\prime} \partial_{\Phi} f+\mathcal{L}_{\Phi}-\square f-\Lambda_{5}$.

Note that, for notational simplicity, we have absorbed the gravitational constant $\kappa_{5}^{2}$ in the expression of the general coupling function $f(\Phi)$.

As was explicitly shown in the Appendix B of [74], not all of Eqs. (7) and (12)-(14) are independent. Therefore, in what follows, we ignore Eq. (7) and work only with the gravitational equations. Of them, Eq. (14) serves to determine the scalar potential in the bulk $V_{B}(\Phi)$. It is Eq. (13) that will provide the solution for the scalar field $\Phi$ once the warp function $A(y)$, the mass function $m(r)$, and the nonminimal coupling function $f(\Phi)$ are determined. For the warp factor, we make the assumption that this is given by the well-known form $A(y)=-k|y|[11,12]$, with $k$ being a positive constant, as this ensures the localization of gravity near the brane. The form of the mass function $m(r)$ readily follows by direct integration of Eq. (12) that leads to the expression

$$
m(r)=M+\Lambda r^{3} / 6,
$$

where $M$ and $\Lambda$ are arbitrary integration constants (the numerical coefficient $1 / 6$ has been introduced for later convenience). Substituting the above form into line element (6) and setting $y=0$, we may easily see that the projectedon-the-brane background is given by the expression

$$
\begin{aligned}
d s_{4}^{2}= & -\left(1-\frac{2 M}{r}-\frac{\Lambda r^{2}}{3}\right) d v^{2}+2 d v d r \\
& +r^{2}\left(d \theta^{2}+\sin ^{2} \theta d \varphi^{2}\right)
\end{aligned}
$$

In [74], we explicitly demonstrated that the above Vaidya form of the four-dimensional line element may be transformed to the usual Schwarzschild-(anti-) de Sitter solution by an appropriate coordinate transformation. Therefore, the arbitrary parameter $M$ is the mass of the black hole that the four-dimensional observer sees and $\Lambda$ the cosmological constant on the brane.

The case of a positive cosmological constant on the brane (i.e., $\Lambda>0$ ) was studied in our previous work [74]; in the context of the present analysis, we focus on the case of a negative four-dimensional cosmological constant $(\Lambda<0)$. Employing the form of the mass function (15) and the exponentially decreasing warp factor ${ }^{2} e^{2 A(y)}=$ $e^{-2 k y}$, Eq. (13) takes the form

$$
\left(\Phi^{\prime}\right)^{2}=-\partial_{y}^{2} f-k \partial_{y} f-\Lambda e^{2 k y} f .
$$

\footnotetext{
${ }^{2}$ We assume a $\mathbf{Z}_{2}$-symmetry in the bulk under the change $y \rightarrow-y$; therefore, henceforth, we focus on the positive $y$-regime.
} 
In the above, we have also used the relations

$$
\partial_{y} f=\Phi^{\prime} \partial_{\Phi} f, \quad \partial_{y}^{2} f=\Phi^{\prime 2} \partial_{\Phi}^{2} f+\Phi^{\prime \prime} \partial_{\Phi} f .
$$

The lhs of Eq. (17) is positive; therefore the same should hold for the rhs, too. Note that, for $\Lambda>0$, Eq. (17) demands that, at least at $y \rightarrow \infty$, the coupling function $f(\Phi)$ should be negative for the scalar field to have a real first derivative there. Indeed, in [74], we presented two analytic solutions of this theory where $f<0$ either far away from our brane or in the entire bulk regime. In contrast, in the present case, where $\Lambda<0$, no such behavior is necessary; thus in order to have a normal gravity over the entire five-dimensional space-time, we assume that $f(\Phi)$ is positive everywhere.

In order to have a physically acceptable behavior, a few more properties should be assigned to the functions $\Phi=$ $\Phi(y)$ and $f=f[\Phi(y)]$. Both functions should, of course, be real and finite in their whole domain and of class $C^{\infty}$. At $y \rightarrow \infty$, both functions should satisfy the following relations; otherwise the finiteness of the theory at infinity cannot be ascertained,

$$
\begin{aligned}
& \lim _{y \rightarrow \infty} \frac{d^{n}[f(y)]}{d y^{n}}=0, \quad \forall n \geq 1, \\
& \lim _{y \rightarrow \infty} \frac{d^{n}[\Phi(y)]}{d y^{n}}=0, \quad \forall n \geq 1 .
\end{aligned}
$$

These constraints guarantee that all components of the energy-momentum tensor $T^{(\Phi) M}{ }_{N}$ will be real and finite everywhere, and, in addition, localized close to our brane. Then, demanding also the finiteness and the vanishing of the rhs of Eq. (17) due to the constraint (20), we conclude that the coupling function $f(y)$ should, at infinity, decrease faster $^{3}$ than $e^{-2 k y}$, i.e., $f(y)$ should be of the form

$$
f(y)=f_{0} e^{g(y)}, \quad\left\{\begin{array}{c}
f_{0}>0 \\
g(y \rightarrow \infty)<-2 k y
\end{array}\right\} .
$$

Consequently, upon integrating Eq. (17), the following expression is obtained for the scalar field:

$\Phi(y)= \pm \sqrt{f_{0}} \int d y e^{\frac{g(y)}{2}} \sqrt{\tilde{\Lambda}^{2} e^{2 k y}-g^{\prime \prime}-g^{\prime 2}-k g^{\prime}}$,

where, for convenience, we have also set $\Lambda=-\tilde{\Lambda}^{2}$. In order to proceed further, we need to determine the exact form of the function $g(y)$. As we are interested in deriving analytical solutions for both functions $f(y)$ and $\Phi(y)$, the

\footnotetext{
${ }^{3}$ Note that allowing the coupling function to vary exactly as $e^{-2 k y}$, i.e., $f(y)=f_{0} e^{-2 k y}$, would lead to a finite, constant value of $\Phi^{\prime 2}$ at infinity, namely, $\Phi_{\infty}^{\prime 2}=-f_{0} \Lambda>0$. This would amount to having a diverging field at the boundary of spacetime but nevertheless finite, constant values for the components of the energy-momentum tensor. We come back to this point later.
}

function $g(y)$ should have a specific form in order to result in a solvable integral on the rhs of Eq. (22). Therefore, we make the following two choices:

$$
\begin{gathered}
g(y)=-\lambda k y, \quad \lambda \in(2,+\infty), \\
g(y)=-\mu^{2} e^{\lambda y}, \quad\left\{\begin{array}{c}
\lambda \in(0,+\infty) \\
\mu \in \Re \backslash\{0\}
\end{array}\right\} .
\end{gathered}
$$

The aforementioned expressions for $g(y)$ ensure that both $f(y)$ and $\Phi(y)$ have the desired properties outlined above and, in addition, lead to analytical solutions. In the following sections, these two different cases are studied separately.

\section{THE SIMPLE EXPONENTIAL CASE}

We start with the simple exponential case (23), and derive first the form of the scalar field and its potential in the bulk. We then study their main characteristics in terms of the free parameters of the model, and finally address the effect of the junction conditions and the form of the effective theory on the brane.

\section{A. The bulk solution}

In this case, we have $f(y)=f_{0} e^{-\lambda k y}$, with $f_{0}>0$ and $\lambda>2$. Then, from Eq. (17), we obtain

$$
\Phi^{\prime 2}(y)=f(y)\left(\tilde{\Lambda}^{2} e^{2 k y}-\lambda^{2} k^{2}+\lambda k^{2}\right) \geq 0 .
$$

For a nonzero and positive $f(y)$, the above inequality demands that the combination inside the brackets should be positive. As this is an increasing function of $y$, it suffices to demand that this holds at the location of the brane, at $y=0$. Then, we obtain the following constraint on the parameters of the theory:

$$
\frac{\tilde{\Lambda}^{2}}{\lambda(\lambda-1) k^{2}}>1
$$

The function $\Phi^{\prime 2}(y)$ could, in principle, be 0 at the point where $\Phi(y)$ has an extremum. However, from Eq. (25), we may easily see that this may happen only at $y_{0}=$ $\frac{1}{2 k} \ln \left(\frac{\lambda(\lambda-1) k^{2}}{\tilde{\Lambda}^{2}}\right)$, which, upon using Eq. (26), turns out to be negative. Therefore, the scalar field does not have any extremum in the whole domain $0 \leq y<\infty$, which in turn means that $\Phi(y)$ is a one-to-one function in the same region. The $\mathbf{Z}_{2}$ symmetry of the extra dimension ensures that this result holds in the region $y<0$ as well. We note this property for later use.

Equation (17) can be rewritten as

$$
\begin{aligned}
\left(\Phi^{\prime}\right)^{2} & =f_{0} \lambda(\lambda-1) k^{2}\left[\frac{\tilde{\Lambda}^{2}}{\lambda(\lambda-1) k^{2}} e^{2 k y}-1\right] e^{-\lambda k y} \\
& =f_{0} \lambda(\lambda-1) k^{2}\left[\frac{\tilde{\Lambda}^{2}}{\lambda(\lambda-1) k^{2}}\right]^{\lambda / 2}(w-1) w^{-\lambda / 2},
\end{aligned}
$$


where we have introduced the new variable $w$ via the definition

$$
w(y) \equiv \frac{\tilde{\Lambda}^{2} e^{2 k y}}{\lambda(\lambda-1) k^{2}} .
$$

Because of the constraint (26), it is obvious that $w(y)$ is greater than unity for all values of the extra coordinate $y$. Then, applying the chain rule to the lhs of Eq. (27) and integrating, we obtain for the scalar field the integral expression

$$
\begin{aligned}
\Phi(w)= & \pm \frac{\sqrt{f_{0} \lambda(\lambda-1)}}{2}\left[\frac{\tilde{\Lambda}^{2}}{\lambda(\lambda-1) k^{2}}\right]^{\lambda / 4} \\
& \times \int d w(w-1)^{\frac{1}{2}} w^{-\frac{\lambda}{4}-1} .
\end{aligned}
$$

In order to evaluate the above integral, we perform a second change of variable; namely, we set $w=1 /(1-z)$. Then,

$$
\begin{aligned}
\int d w(w-1)^{\frac{1}{2}} w^{-\frac{\lambda}{4}-1} & =\int d z z^{\frac{1}{2}}(1-z)^{\frac{\lambda}{4}-\frac{3}{2}} \\
& =\int_{0}^{z} d t t^{\frac{1}{2}}(1-t)^{\frac{\lambda}{4}-\frac{3}{2}}+C_{1},
\end{aligned}
$$

where an arbitrary constant $C_{1}$ has been introduced in order to set the lower boundary value of the integral equal to 0 . Finally, by employing the rescaled variable $t^{\prime}=t / z$, the above integral takes its final form

$$
\begin{aligned}
& z^{\frac{3}{2}} \int_{0}^{1} d t^{\prime} t^{\prime \frac{1}{2}}\left(1-z t^{\prime}\right)^{\frac{\lambda}{4}-\frac{3}{2}}+C_{1} \\
& \quad=\frac{2}{3}\left(\frac{w-1}{w}\right)^{3 / 2}{ }_{2} F_{1}\left(\frac{3}{2}-\frac{\lambda}{4}, \frac{3}{2} ; \frac{5}{2} ; \frac{w-1}{w}\right)+C_{1},
\end{aligned}
$$

where we used the integral representation of the hypergeometric function [92]

$$
\begin{aligned}
{ }_{2} F_{1}(a, b ; c ; z)= & \frac{\Gamma(c)}{\Gamma(b) \Gamma(c-b)} \\
& \times \int_{0}^{1} d t^{\prime} t^{\prime b-1}\left(1-t^{\prime}\right)^{c-b-1}\left(1-z t^{\prime}\right)^{-a}, \\
& \operatorname{Re}(c)>\operatorname{Re}(b)>0 .
\end{aligned}
$$

We now observe that $\Phi$ appears in the field equations (7), (13), and (14) only through the coupling function $f(\Phi)$ and the bulk potential $V_{B}(\Phi)$. Therefore, any shift in the value of the scalar field by an arbitrary constant would result in a change in the value of $f$ by a constant amount that could nevertheless be reabsorbed in the redefinition of the value of the arbitrary coefficient $f_{0}$; the value of the bulk potential $V_{B}$ would also change by a constant amount but this could again be reabsorbed in the value of the arbitrary bulk cosmological constant $\Lambda_{5}$. Because of this translation symmetry with respect to the value of the scalar field $\Phi(y)$, we may set the arbitrary constant $C_{1}$ in Eq. (31) equal to 0 . This brings the solution for the scalar field into its final form,

$$
\begin{aligned}
\Phi_{ \pm}(y)= & \pm \frac{\sqrt{f_{0} \lambda(\lambda-1)}}{3}\left[\frac{\tilde{\Lambda}^{2}}{\lambda(\lambda-1) k^{2}}\right]^{\lambda / 4}\left[\frac{w(y)-1}{w(y)}\right]^{3 / 2} \\
& \times{ }_{2} F_{1}\left(\frac{3}{2}-\frac{\lambda}{4}, \frac{3}{2} ; \frac{5}{2} ; \frac{q w(y)-1}{w(y)}\right) .
\end{aligned}
$$

Although the function $w(y)$ is greater than unity for all values of the extra dimension $y$, the argument $z=\frac{w-1}{w}$ of the hypergeometric function in the previous relation is always positive and smaller than unity. Hence, we may use the well-known expansion for the hypergeometric function in power series,

${ }_{2} F_{1}(a, b ; c ; z)={ }_{2} F_{1}(b, a ; c ; z)=\sum_{n=0}^{\infty} \frac{a^{(n)} b^{(n)}}{c^{(n)}} \frac{z^{n}}{n !}$,

where $|z|<1$, and the quantities of the form $q^{(n)}$ denote (rising) Pochhammer symbols, namely,

$q^{(n)}=\frac{\Gamma(q+n)}{\Gamma(q)}=\left\{\begin{array}{ll}q(q+1) \cdots(q+n-1), & n>0 \\ 1, & n=0\end{array}\right.$.

Thus, we find

$$
\begin{aligned}
{ }_{2} F_{1}\left(\frac{3}{2}-\frac{\lambda}{4}, \frac{3}{2} ; \frac{5}{2} ; \frac{w-1}{w}\right)= & \sum_{n=0}^{\infty} \frac{\Gamma\left(\frac{3}{2}-\frac{\lambda}{4}+n\right)}{\Gamma\left(\frac{3}{2}-\frac{\lambda}{4}\right)} \frac{3}{(2 n+3) n !} \\
& \times\left(\frac{w-1}{w}\right)^{n}
\end{aligned}
$$

where we have also used the property $\Gamma(1+z)=z \Gamma(z)$. There are two interesting categories of values for the parameter $\lambda$ that lead to simple and elegant expressions for the hypergeometric function and subsequently for the scalar field. These are $\lambda=2(1+2 q)$ and $\lambda=4 q$, where $q$ is any positive integer. Let us examine each case separately.

(i) If $\lambda=2(1+2 q)$ with $q \in \mathbb{Z}^{>}$, then, from Eq. (36), we have 


$$
\begin{aligned}
& { }_{2} F_{1}\left(\frac{3}{2}-\frac{\lambda}{4}, \frac{3}{2} ; \frac{5}{2} ; \frac{w-1}{w}\right) \\
& =\sum_{n=0}^{\infty}(1-q)^{(n)} \frac{3}{(2 n+3) n !}\left(\frac{w-1}{w}\right)^{n} \\
& =\left\{\begin{array}{ll}
1, & q=1 \\
1+\sum_{n=1}^{q-1} \frac{3(-q+1)(-q+2) \cdots(-q+n)}{(2 n+3) n !}\left(\frac{w-1}{w}\right)^{n}, & q>1
\end{array}\right\} .
\end{aligned}
$$

In the second line of the above expression, the upper limit of the sum has been changed from $\infty$ to $q-1$ since, for $q$ and $n$ positive, the sum is trivial for any value of $n$ equal or higher than $q$ due to the factor $(-q+n)$. As indicative cases, we present below the form of the scalar field for $^{4} q=1$ (i.e., $\lambda=6$ ),

$$
\Phi_{ \pm}(y)= \pm \frac{\sqrt{f_{0}}}{90}\left(\frac{\tilde{\Lambda}^{2}}{k^{2}}\right)^{3 / 2}\left(\frac{w-1}{w}\right)^{3 / 2},
$$

and $q=2$ (i.e., $\lambda=10$ ),

$$
\begin{aligned}
\Phi_{ \pm}(y)= & \pm \frac{\sqrt{f_{0}}}{3 \times 90^{2}}\left(\frac{\tilde{\Lambda}^{2}}{k^{2}}\right)^{5 / 2}\left(\frac{w-1}{w}\right)^{3 / 2} \\
& \times\left[1-\frac{3}{5}\left(\frac{w-1}{w}\right)\right] .
\end{aligned}
$$

The above expressions follow easily by using Eqs. (33) and (37) and substituting the aforementioned values of the parameter $q$.

(ii) If $\lambda=4 q$ with $q \in \mathbb{Z}^{>}$, we can always express the hypergeometric function in Eq. (33) in terms of elementary functions, namely, $\arcsin \left(\sqrt{\frac{w-1}{w}}\right)$, square roots, and powers of the argument $\frac{w-1}{w}$. The process that one follows to obtain this expression is presented in detail in Appendix B. Thus, for $\lambda=4$ (i.e., $q=1$ ), using Eq. (B3), we may straightforwardly write

$$
\begin{aligned}
\Phi_{ \pm}(y)= & \pm \frac{\sqrt{f_{0}}}{4 \sqrt{3}} \frac{\tilde{\Lambda}^{2}}{k^{2}}\left(\frac{w-1}{w}\right)^{1 / 2} \\
& \times\left[\sqrt{\frac{w}{w-1}} \arcsin \left(\sqrt{\frac{w-1}{w}}\right)-\sqrt{\frac{1}{w}} .\right.
\end{aligned}
$$

\footnotetext{
${ }^{4}$ For completeness, we present here also the solution for the limiting case with $q=0$ (i.e., for $\lambda=2$ ); this has the form

$$
\Phi_{ \pm}(y)= \pm \sqrt{f_{0}} \sqrt{\frac{\tilde{\Lambda}^{2}}{k^{2}}}\left[\operatorname{arctanh}\left(\sqrt{\frac{w-1}{w}}\right)-\sqrt{\frac{w-1}{w}}\right] .
$$

Although the field diverges at infinity-see footnote 2 - the components of the energy-momentum tensor exhibit a regular behavior as we comment later.
}

For larger values of $\lambda$ (i.e., for $q=1+\ell$, with $\ell \in \mathbb{Z}^{>}$), we should use instead Eq. (B11) together with the constraint (B12). The latter, as outlined in Appendix B, reduces to a set of linear equations that determine the unknown coefficients $\alpha, \beta_{1}, \ldots, \beta_{\ell}$. For example, for $\ell=1$, the set of equations that follow from Eq. (B12) is

$$
\left\{2 \alpha-\beta_{1}=0, \quad 2 \alpha+3 \beta_{1}=3\right\},
$$

leading to the values $\alpha=3 / 8$ and $\beta=3 / 4$. Then, after substituting these in Eq. (B11), the solution for $q=2$, or equivalently for $\lambda=8$, follows from Eq. (33) and has the form

$$
\begin{aligned}
\Phi_{ \pm}(y)= & \pm \frac{\sqrt{f_{0}}}{896 \sqrt{14}} \frac{\tilde{\Lambda}^{4}}{k^{4}}\left(\frac{w-1}{w}\right)^{1 / 2} \\
& \times\left[\sqrt{\frac{w}{w-1}} \arcsin \left(\sqrt{\frac{w-1}{w}}\right)\right. \\
& \left.+\sqrt{\frac{1}{w}}\left(1-\frac{2}{w}\right)\right] .
\end{aligned}
$$

Solutions for larger values of $q$, and thus of $\lambda$, may be derived in the same way in terms again of analytic, elementary functions.

In all the above, particular expressions for the scalar field $\Phi_{ \pm}$, which follow for specific values of the parameter $\lambda$, the dependence on the extra coordinate $y$ is easily made explicit by employing Eq. (28). Also, for all other values of $\lambda \in \mathbb{R}^{>2}$, which do not fall in the aforementioned categories, the scalar field may still be expressed in terms of the hypergeometric function through Eqs. (33) and (36).

Let us now investigate the physical characteristics of the solutions we have derived. In Fig. 1(a), we depict the form of the warp factor $e^{-2 k|y|}$ and the coupling function $f(y)=$ $f_{0} e^{-\lambda k|y|}$ in terms of the coordinate $y$ along the fifth dimension, for $f_{0}=1, k=1$ and $\lambda=5$. The warp factor exhibits the anticipated localization close to the brane while the nonminimal coupling function mimics this behavior by decreasing exponentially fast away from the brane and reducing to 0 at the boundary of spacetime. In fact, the larger the parameter $\lambda$, the faster the decrease rate of $f$ is; thus by increasing $\lambda$, the nonminimal coupling of the scalar field to gravity is effectively localized closer to the brane.

Figure 1(b) depicts the scalar field $\Phi_{+}(y)$ for different values of the parameter $\lambda$ and for $f_{0}=1, k=1$, $\tilde{\Lambda}^{2} / k^{2}=100$. It is straightforward to deduce from Fig. 1(b) that the scalar field $\Phi_{+}(y)$ exhibits a reverse behavior, compared to $f(y)$, by increasing away from the brane and adopting a constant, nonvanishing value at the boundary of spacetime. Note that, as $\lambda$ increases, the scalar field reaches this constant asymptotic value faster; that is, a more localized coupling function keeps also the nontrivial profile of the scalar field closer to the brane. Overall, for $\lambda>2$, the 


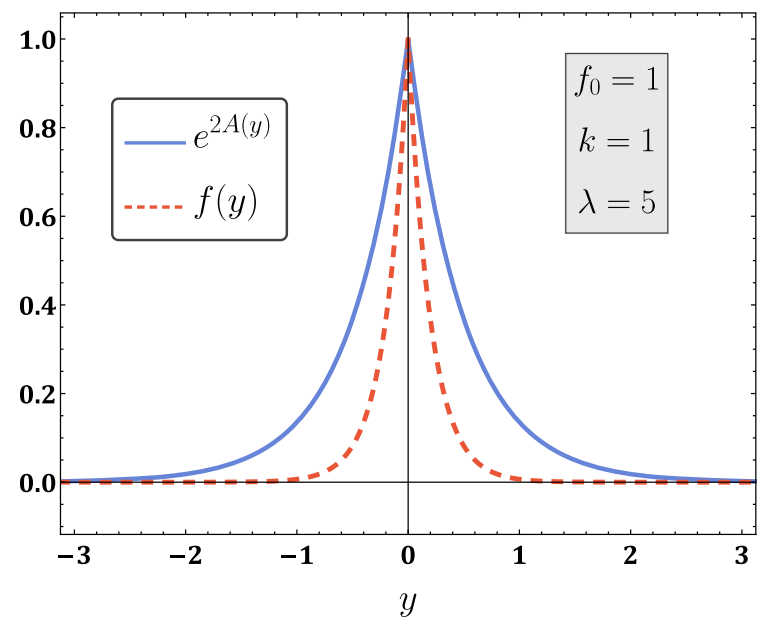

(a)

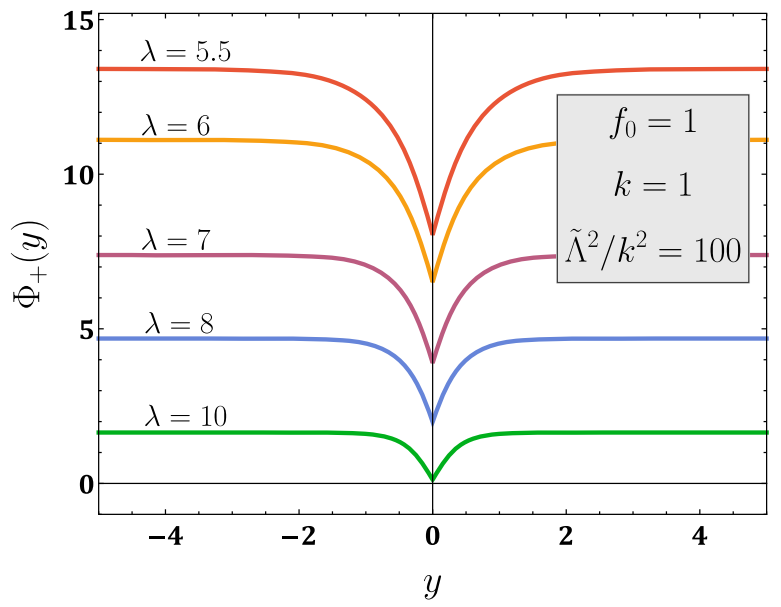

(b)

FIG. 1. (a) The warp factor $e^{2 A(y)}=e^{-2 k|y|}$ and the coupling function $f(y)=f_{0} e^{-\lambda k|y|}$ in terms of the coordinate $y$, for $f_{0}=1, k=1$, $\lambda=5$. (b) The scalar field $\Phi_{+}(y)$ also in terms of the coordinate $y$, for $f_{0}=1, k=1, \tilde{\Lambda}^{2} / k^{2}=100$ and $\lambda=5.5,6,7,8,10$ (from top to bottom).

scalar field presents a well-defined profile over the entire extra dimension in accordance with the desired properties set in the previous section. In Fig. 1(b), we chose to plot $\Phi_{+}(y)$; i.e., we chose the positive sign in Eq. (33) for the expression of the scalar field. A second class of solutions exists for $\Phi=\Phi_{-}$, with the only difference being Fig. 1(b) becoming its mirror image with respect to the horizontal axis. The sign of the scalar field, however, does not affect either the potential $V_{B}(y)$ or the components of the energymomentum tensor, as we soon see. Finally, let us emphasize the fact that, as Fig. 1(b) reveals, the qualitative behavior of the scalar field in terms of the parameter $\lambda$ remains unchanged. This holds despite the fact that the value of $\lambda$ does affect the exact, analytic expression of the scalar field, as we have shown in detail above; we may thus conclude that solutions emerging for nonminimal coupling functions of a simple exponential form, differing only in the value of the parameter $\lambda$, i.e., in the decrease rate of $f$ with $y$, lead to a class of black-string solutions with the same qualitative characteristics.

The potential of the field $V_{B}$ in the bulk can be determined from Eq. (14): substituting the functions $m(r)$ and $A(y)$, we obtain

$V_{B}=-\Lambda_{5}-\frac{1}{2} \Phi^{\prime 2}+3 k \partial_{y} f-\partial_{y}^{2} f-f\left(6 k^{2}-\Lambda e^{2 k y}\right)$,

where we have also used the relations (18). Note that the bulk potential is indeed insensitive to the sign of $\Phi_{ \pm}$that enters the above expression through $\Phi^{\prime 2}$. If we also employ Eq. (17) to substitute $\Phi^{\prime 2}$, and use the exponential form for $f(y)$, the following expression readily follows for the potential $V_{B}$ in terms of the extra dimension $y$ :
$V_{B}(y)=-\Lambda_{5}-\frac{k^{2} f_{0}}{2} e^{-\lambda k y}\left(12+7 \lambda+\lambda^{2}+\frac{3 \tilde{\Lambda}^{2}}{k^{2}} e^{2 k y}\right)$.

We observe that the combination $V_{B}(y)+\Lambda_{5}$, which appears in the action (1) as well as in the components of the total energy-momentum tensor as we shortly see, is always negative definite. This combination, even for $\Lambda_{5}=0$, may therefore provide by itself the negative distribution of energy in the bulk that is necessary for the support of the AdS spacetime and the localization of gravity. A similar result was derived in [74] where the case of a positive cosmological constant $\Lambda$ on the brane was considered: there, the positive $\Lambda$ added a positive contribution to the value of $V_{B}$ that was thus decreased in absolute value, while here the negative $\Lambda$ gives an extra boost to the negative value of $V_{B}$. The profile of the bulk potential of the scalar field is depicted in Fig. 2(a) for $f_{0}=1 / 5, k=1, \tilde{\Lambda}^{2} / k^{2}=9$, and $\lambda=2.5$. The potential is everywhere finite and remains localized close to the brane. For all values of $\lambda>2$, it goes to 0 with an exponential decay rate that increases with $\lambda$. The vanishing of $V_{B}$ at the boundary of space-time, together with the similar behavior of the coupling function $f$ in the same regime and the constant value that the scalar field assumes there, points to the conclusion that the nonminimally coupled scalar field, after serving its purpose of localizing gravity close to the brane, completely disappears leaving behind a fivedimensional Minkowski space-time. Only, for $\lambda=2$, an asymptotic bulk cosmological constant equal to $-3 f_{0} \tilde{\Lambda}^{2} / 2$ remains, thus leading to an asymptotically AdS space-time, but only by paying the price of a diverging scalar field at infinity. 


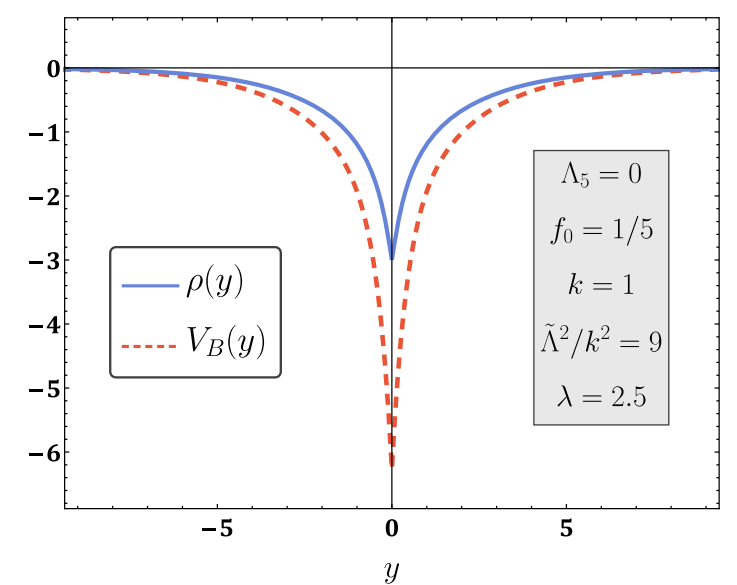

(a)

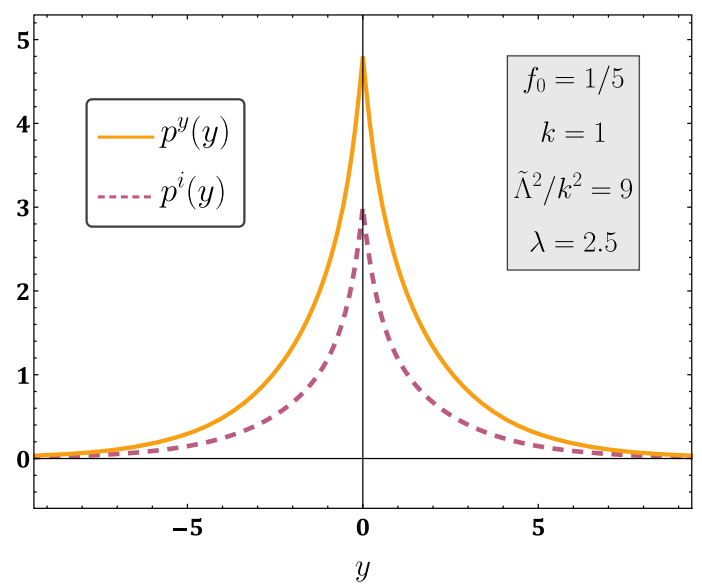

(b)

FIG. 2. (a) The scalar potential $V_{B}$ and energy density $\rho$ of the system, and (b) the pressure components $p^{y}$ and $p^{i}$ in terms of the coordinate $y$.

Finally, we may compute the components of the energymomentum tensor of the theory in the bulk. These follow by employing Eqs. (5) and (9). Using also the relations $\rho=-T_{0}^{0}, p^{i}=T^{i}{ }_{i}$, and $p^{y}=T^{y}{ }_{y}$, we find the results

$$
\begin{gathered}
\rho=-p^{i}=\frac{1}{2} \Phi^{\prime 2}+V_{B}+\Lambda_{5}-3 k \partial_{y} f+\partial_{y}^{2} f, \\
p^{y}=\frac{1}{2} \Phi^{\prime 2}-V_{B}-\Lambda_{5}+4 k \partial_{y} f .
\end{gathered}
$$

Substituting $V_{B}$ from Eq. (43) and the form of the coupling function, we finally obtain the following explicit expressions:

$$
\begin{gathered}
\rho=-p^{i}=-f_{0} e^{-\lambda k y}\left(6 k^{2}+\tilde{\Lambda}^{2} e^{2 k y}\right), \\
p^{y}=f_{0} e^{-\lambda k y}\left(6 k^{2}+2 \tilde{\Lambda}^{2} e^{2 k y}\right)
\end{gathered}
$$

We present the behavior of the energy density $\rho$ in Fig. 2(a) and of the pressure components $p^{i}$ and $p^{y}$ in Fig. 2(b) with respect to the extra dimension $y$. Both figures have the same values for the parameters of the model to allow for an easy comparison. The energy density is negative definite throughout the bulk, due to the negative value of the scalar potential discussed above, in order to support the pseudoAdS space-time and the exponentially falling warp factor. The spacelike pressure components $p^{i}$ satisfy the relation $p^{i}=-\rho$, a remnant of the equation of state of a true cosmological constant. The fifth pressure component $p^{y}$ is also positive but larger than $p^{i}$ due to the factor of 2 in front of $\tilde{\Lambda}^{2}$ in Eq. (48). All components present a well-defined profile throughout the bulk and vanish exponentially fast away from the brane for all $\lambda>2$. The aforementioned behavior remains qualitatively the same for all values of the parameters of the model.

\section{B. Junction conditions and effective theory}

We now turn our attention to the junction conditions that must be incorporated in the model due to the presence of the brane at $y=0$. We assume that the energy content of the brane is given by the combination $\sigma+V_{b}(\Phi)$, where $\sigma$ is the constant self-energy of the brane and $V_{b}(\Phi)$ an interaction term of the bulk scalar field with the brane. This energy content is assumed to arise only at a single point along the extra dimension, i.e., along our brane at $y=0$, and thus it creates a discontinuity in the second derivatives of the warp factor, the coupling function, and the scalar field at the location of the brane. Following the standard literature [93], we write $A^{\prime \prime}=\hat{A}^{\prime \prime}+\left[A^{\prime}\right] \delta(y), f^{\prime \prime}=\hat{f}^{\prime \prime}+$ $\left[f^{\prime}\right] \delta(y)$ and $\Phi^{\prime \prime}=\hat{\Phi}^{\prime \prime}+\left[\Phi^{\prime}\right] \delta(y)$, where the hat quantities denote the distributional (i.e., regular) parts of the second derivatives and $[\cdots]$ stand for the discontinuities of the corresponding first derivatives across the brane. Then, going back to the field equations (7) and (13), we reintroduce the delta-function terms that we omitted while working in the bulk. If we then match the coefficients of the delta-function terms appearing in Eqs. (7) and (13), we obtain the following two conditions,

$$
\begin{gathered}
{\left[\Phi^{\prime}\right]=4\left[A^{\prime}\right] \partial_{\Phi} f+\partial_{\Phi} V_{b},} \\
3 f(y)\left[A^{\prime}\right]=-\left[f^{\prime}\right]-\left(\sigma+V_{b}\right),
\end{gathered}
$$

respectively, where all quantities are evaluated at $y=0^{+}$. Using the expressions for the warp function $A(y)=-k|y|$ and the coupling function $f(y)=f_{0} e^{-\lambda k|y|}$ in Eq. (50), and making use of the assumed $\mathbf{Z}_{2}$ symmetry in the bulk, we readily obtain the constraint

$$
\sigma+\left.V_{b}(\Phi)\right|_{y=0}=2 k f_{0}(\lambda+3)
$$

We note that the combination of parameters on the rhs of the above equation is positive definite; therefore, the total 
energy density of our brane is always positive. The above constraint may be used to determine the value of the warpfactor parameter $k$ in terms of the fundamental quantities of the brane tension $\sigma$ and the scalar-field parameters $\left(f_{0}\right.$, $\left.\lambda, V_{b}\right)$. We thus observe that, once we decide the form of the nonminimal coupling function, the warping gets stronger the larger the interaction term of the scalar field with the brane is.

In order to evaluate the first constraint (49), we write that $\partial_{\Phi} f=\partial_{y} f / \Phi^{\prime}$ and $\partial_{\Phi} V_{b}=\partial_{y} V_{b} / \Phi^{\prime}$. We are allowed to do this since, as we showed in Sec. II, the function $\Phi(y)$ does not possess any extrema in the bulk; therefore, $\Phi^{\prime}(y)$ never vanishes. Then, multiplying both sides of Eq. (49) by $\Phi^{\prime}$ and using Eq. (17), we obtain the condition

$$
\left.\partial_{y} V_{b}\right|_{y=0}=2 f_{0}\left[\tilde{\Lambda}^{2}-k^{2} \lambda(\lambda+3)\right] .
$$

This second constraint may be used in a twofold way: for a nontrivial interaction term $V_{b}$, it may serve to determine an independent parameter in its expression; alternatively, under the condition that $V_{b}=$ const. and thus $\partial_{y} V_{b}=0$, it may determine the value of the effective cosmological constant on the brane to be $\Lambda=-\tilde{\Lambda}^{2}=-k^{2} \lambda(\lambda+3)$, a value that is absolutely compatible with the original constraint (26) that should hold on the brane.

Let us finally address the issue of the effective theory on the brane. For this, we need to derive the four-dimensional effective action by integrating the complete fivedimensional one $S=S_{B}+S_{b r}$, over the fifth coordinate $y$. Before we proceed though, we present the explicit forms of the five-dimensional curvature invariants whose general form for the metric ansatz (6) is given in Appendix A. Substituting the mass function (15) and the warp function $A(y)=-k|y|$ in these expressions, we obtain

$$
\begin{aligned}
R= & -20 k^{2}+4 \Lambda e^{2 k|y|}, \\
R_{M N} R^{M N}= & 80 k^{4}-32 k^{2} \Lambda e^{2 k|y|}+4 \Lambda^{2} e^{4 k|y|}, \\
R_{M N K L} R^{M N K L}= & 40 k^{4}-16 k^{2} \Lambda e^{2 k|y|} \\
& +\frac{8 \Lambda^{2} e^{4 k|y|}}{3}+\frac{48 M^{2} e^{4 k|y|}}{r^{6}},
\end{aligned}
$$

where $\Lambda=-\tilde{\Lambda}^{2}$ is the negative constant appearing in the projected-on-the-brane gravitational background (16). The above expressions are valid in the domain $y \in(-\infty, 0) \cup$ $(0, \infty)$, i.e., throughout the bulk where the second derivative of the warp factor equals 0 . Note that, by keeping $M$ in the form of the mass function (15), the obtained solutions describe clearly a black string with its singularity at $r=0$ extending along the extra dimension. Setting, however, $M=0$, we obtain solutions that are maximally symmetric on the brane and possess only a true singularity at the boundary of the bulk space-time where $y \rightarrow \infty$.

This latter singularity was not present in the case of the black-string solution of Ref. [16], when $M=0$. We note that the singular terms in Eq. (53) are directly related to the integration constant $\Lambda$ that, as we soon see, is interpreted as the cosmological constant on the brane. Our scalartensor theory allows for solutions with nonzero cosmological constant on the brane while the model employed in Ref. [16] assumed the Randall-Sundrum fine-tuning between the bulk cosmological constant and the brane tension to ensure a flat brane. If we also set $\Lambda=0$ in our analysis, this additional singularity disappears and we recover a regular AdS space-time as in [16]. However, we consider the presence of a nonzero cosmological constant on the brane as an important feature of the solutions, the effect of which has not been adequately studied in the literature. To this end, our analysis reveals that a nonzero $\Lambda$ on the brane is accompanied by a singularity in the bulk, located at an infinite coordinate distance from our brane. ${ }^{5}$

Returning to the four-dimensional effective theory, we first calculate the effective gravitational scale $M_{\mathrm{Pl}}^{2}$ on the brane. To this end, by employing the first of Eqs. (53), we may write $R=-20 k^{2}+R^{(4)} e^{2 k|y|}$, where $R^{(4)}=4 \Lambda$ is the four-dimensional scalar curvature, which may easily be computed from the projected-on-the-brane line element (16). Hence, the term from the complete action $S=S_{B}+$ $S_{b r}$ that is relevant for the evaluation of the effective gravitational constant is the following:

$$
S \supset \int d^{4} x d y \sqrt{-g^{(5)}} \frac{f(\Phi)}{2} e^{2 k|y|} R^{(4)}
$$

Then, using also that $\sqrt{-g^{(5)}}=e^{-4 k|y|} \sqrt{-g^{(b r)}}$, where $g_{\mu \nu}^{(b r)}$ is the metric tensor of the projected on the brane space-time, the four-dimensional, effective gravitational constant is given by the integral

$$
\begin{aligned}
\frac{1}{\kappa_{4}^{2}} & \equiv 2 \int_{0}^{\infty} d y e^{-2 k y} f(y)=2 f_{0} \int_{0}^{\infty} d y e^{-2 k y} e^{-\lambda k y} \\
& =\frac{2 f_{0}}{k(\lambda+2)} .
\end{aligned}
$$

Since $1 / \kappa_{4}^{2}=M_{\mathrm{Pl}}^{2} /(8 \pi)$, we obtain

$$
M_{\mathrm{Pl}}^{2}=\frac{16 \pi f_{0}}{k(\lambda+2)}=\frac{32 \pi f_{0}^{2}}{\left.\left(\sigma+V_{b}\right)\right|_{y=0}} \frac{(\lambda+3)}{(\lambda+2)} .
$$

In the last expression above, we have replaced the warpfactor parameter $k$ from Eq. (51). We first note that the integral in Eq. (55) is finite; therefore, there is no need for

\footnotetext{
${ }^{5}$ The presence of this singularity does not affect the remaining features of the solution, such as the scalar-field configuration, the warping of space-time, or the effective theory on the branehowever, if desired, it could easily be shielded by the introduction of a second brane.
} 
the introduction of a second brane in the model (unless one wishes to shield the singularity at the boundary of spacetime by introducing a second brane). Also, according to the above result, the effective gravity scale on the brane $M_{\mathrm{Pl}}^{2}$ is determined by the ratio $f_{0} / k$. Taking into account that $f_{0}$ has units of $[M]^{3}$, after the absorption of $1 / \kappa_{5}^{2}$ in its value, and thus plays the role of the fundamental energy scale $M_{*}^{3}$, the relation between the fundamental and the effective gravity scales turns out to be almost the same as the corresponding one in the Randall-Sundrum II model [12]. The difference between the magnitudes of $M_{*}$ and $M_{\mathrm{Pl}}$ is determined by the combination $f_{0} /\left.\left(\sigma+V_{b}\right)\right|_{y=0}$ (note that $\lambda$ plays virtually no role). Therefore, if a low-gravity scale is desired, i.e., a low $f_{0}$, then the total energy density of the brane should be minimized. This may be realized via the presence of a large, negative interaction term $V_{b}$ for the scalar field that would result in a small, yet positive as dictated by Eq. (51), value for the combination $\left.\left(\sigma+V_{b}\right)\right|_{y=0}$.

To complete our study of the effective theory on the brane, we finally compute the effective cosmological constant. Since the scalar field $\Phi$ is only $y$ dependent, the effective theory contains no dynamical d.o.f. Therefore, the integral of all the remaining terms of the five-dimensional action $S=S_{B}+S_{b r}$, apart from the one appearing in Eq. (54), yields the effective cosmological constant on the brane. Because of the existence of the brane, which acts as a boundary for the five-dimensional space-time, the bulk integral must be supplemented by the source term of the brane as well as the Gibbons-Hawking term [94]. In total, we have

$$
\begin{aligned}
-\Lambda_{4} & =\int_{-\infty}^{\infty} d y e^{-4 k|y|}\left[-10 k^{2} f(y)-\Lambda_{5}-\frac{1}{2} \Phi^{\prime 2}-V_{B}(y)+\left.f(y)\left(-4 A^{\prime \prime}\right)\right|_{y=0}-\left[\sigma+V_{b}(\Phi)\right] \delta(y)\right] \\
& =2 \int_{0}^{\infty} d y e^{-4 k y}\left[-10 k^{2} f(y)-\Lambda_{5}-\frac{1}{2} \Phi^{\prime 2}-V_{B}(y)\right]+8 k f(0)-\left[\sigma+V_{b}(\Phi)\right]_{y=0} .
\end{aligned}
$$

Substituting the expressions for the coupling function and the bulk potential of the scalar field, and employing the junction condition (51), we finally obtain the result

$$
\Lambda_{4}=-\frac{2 f_{0} \tilde{\Lambda}^{2}}{k(\lambda+2)}=\frac{\Lambda}{\kappa_{4}^{2}} .
$$

As expected, the constant of integration $\Lambda$ appearing in the form of the mass function (15), and in the projected-on-thebrane line element (16) is indeed the four-dimensional cosmological constant $\Lambda_{4}$, multiplied by $\kappa_{4}^{2}$, as the inverse Vaidya coordinate transformation on the brane had demonstrated [74].

\section{THE DOUBLE EXPONENTIAL CASE}

We proceed to the alternative form of the nonminimal coupling function given in Eq. (24). As in the previous section, the focus is on the derivation of analytic solutions of the field equations and the study of the characteristics of the resulting solutions both in the bulk and on the brane.

\section{A. The bulk solution}

In this case, the coupling function has the form $f(y)=f_{0} e^{-\mu^{2} e^{\lambda y}}$, with $\lambda$ being any positive real number and $\mu$ a real, nonvanishing number. Substituting the function $g(y)=-\mu^{2} e^{\lambda y}$ in the expression of the scalar field $\Phi(y)$ given by Eq. (22), we obtain the integral expression

$$
\begin{aligned}
\Phi_{ \pm}(y)= & \pm \sqrt{f_{0}} \int d y \exp \left(-\frac{\mu^{2}}{2} e^{\lambda y}\right) \\
& \times \sqrt{\tilde{\Lambda}^{2} e^{2 k y}+\mu^{2} \lambda(\lambda+k) e^{\lambda y}-\mu^{4} \lambda^{2} e^{2 \lambda y}} .
\end{aligned}
$$

In general, the above integral does not have an analytic solution. However, if one chooses appropriate values for the parameters $\mu^{2}$ and $\lambda$, the quantity under the square root can be expressed as a perfect square and the integral becomes solvable. To this end, we can rewrite the aforementioned quantity as

$$
\begin{aligned}
& \tilde{\Lambda}^{2} e^{2 k y}+\mu^{2} \lambda(\lambda+k) e^{\lambda y}-\mu^{4} \lambda^{2} e^{2 \lambda y} \\
& \quad=\left(\sqrt{\tilde{\Lambda}^{2}} e^{k y}-\sqrt{\mu^{2} \lambda(\lambda+k)} e^{\lambda y / 2}\right)^{2},
\end{aligned}
$$

provided that the following conditions are imposed:

$$
k+\frac{\lambda}{2}=2 \lambda, \quad 2 \sqrt{\tilde{\Lambda}^{2} \mu^{2} \lambda(\lambda+k)}=\mu^{4} \lambda^{2} .
$$

These lead to the unique values

$$
\lambda=\frac{2 k}{3}, \quad \mu^{2}=\left(\frac{45}{2} \frac{\tilde{\Lambda}^{2}}{k^{2}}\right)^{\frac{1}{3}},
$$

for the $\lambda$ and $\mu$ parameters. Using the form of the coupling function in Eq. (17), and substituting the above values for $\lambda$ and $\mu^{2}$ in the result, we are led to 


$$
\begin{aligned}
\Phi^{2}(y) & =f(y)\left[\tilde{\Lambda}^{2} e^{2 k y}+\mu^{2} \lambda(\lambda+k) e^{\lambda y}-\mu^{4} \lambda^{2} e^{2 \lambda y}\right] \\
& =\frac{2 k^{2} \mu^{2}}{45} f(y) e^{\frac{2 k y}{3}}\left(\mu^{2} e^{\frac{2 k y}{3}}-5\right)^{2} .
\end{aligned}
$$

The above equation can provide important information on the form of the scalar field in the bulk even before the explicit integration in Eq. (59) is performed. To start with, since $f(y)>0$ for all $y>0$, the rhs of the above relation is automatically positive definite; therefore, no additional constraint on the parameters of the theory follows by demanding the positivity of $\Phi^{\prime 2}$. The value of the parameter $\mu^{2}$ though affects significantly the profile of the scalar field along the extra dimension. In particular, if the value of $\mu^{2}$ is lower than 5, then the first derivative of the scalar field becomes 0 at $y_{0}=\frac{3}{2 k} \ln \left(\frac{5}{\mu^{2}}\right)$. If the value of $\mu^{2}$ is exactly 5 , then the first derivative of the scalar field is 0 at $y=0$. Finally, if $\mu^{2}$ is greater than 5 , then the first derivative of the scalar field does not vanish anywhere in the bulk. In summary,

$$
\left\{\begin{array}{lll}
\mu^{2}<5: & \Phi^{\prime}\left(y_{0}\right)=0, & y_{0}=\frac{3}{2 k} \ln \left(\frac{5}{\mu^{2}}\right), \\
\mu^{2}=5: & \Phi^{\prime}(0)=0, & \\
\mu^{2}>5: & \Phi^{\prime}(y) \neq 0, & \forall y>0 .
\end{array}\right\}
$$

Taking however the square root of Eq. (63), we obtain for $\Phi^{\prime}(y)$ the expression

$\Phi^{\prime}(y)= \pm k \sqrt{\frac{2 f_{0} \mu^{2}}{45}} \exp \left(-\frac{\mu^{2}}{2} e^{\frac{2 k y}{3}}+\frac{k y}{3}\right)\left|\mu^{2} e^{\frac{2 k y}{3}}-5\right|$.

We thus conclude that $\Phi^{\prime}(y)$ retains a specific sign throughout the bulk, even in the case where $\mu^{2}<5$; therefore, the point $y=y_{0}$ is not an extremum [where $\Phi^{\prime}(y)$ owes to change its sign] but rather an inflection point. As a result, $\Phi(y)$ is a monotonic function throughout the bulk, for all values of $\mu^{2}$, and thus it is a one-to-one function in the whole region $y>0$ (as well as in the $y<0$ region due to the $\mathbf{Z}_{2}$ symmetry).

The above behavior also affects the way that one should proceed in order to find the solution for the scalar field $\Phi$. For $\mu^{2} \geq 5$, the quantity inside the absolute value in Eq. (65) is positive and nonvanishing for all values $y>0$; thus, the solution for $\Phi(y)$, at every point in the bulk, follows by directly integrating Eq. (65). Then, we obtain

$$
\Phi_{ \pm}(y)= \pm[\mathcal{I}(y)-\mathcal{I}(0)]
$$

where we have defined $\mathcal{I}(y)$ as

$$
\begin{aligned}
\mathcal{I}(y) \equiv & -\sqrt{\frac{f_{0}}{5}}\left[\sqrt{2 \mu^{2}} \exp \left(\frac{k y}{3}-\frac{1}{2} \mu^{2} e^{\frac{2 k y}{3}}\right)\right. \\
& \left.+4 \sqrt{\pi} \operatorname{erf}\left(\sqrt{\frac{\mu^{2}}{2}} e^{\frac{k y}{3}}\right)\right],
\end{aligned}
$$

and $\operatorname{erf}(z)$ is the error function

$$
\operatorname{erf}(z)=\frac{2}{\sqrt{\pi}} \int_{0}^{z} e^{-t^{2}} d t=\frac{2}{\sqrt{\pi}} \sum_{n=0}^{\infty} \frac{(-1)^{n} z^{2 n+1}}{n !(2 n+1)} .
$$

On the other hand, for $\mu^{2}<5$, we need to address separately the cases where the solution for $\Phi(y)$ is found at a point in the bulk with $y \leq y_{0}$ or at a point beyond the inflection point with $y>y_{0}$. In the first case, apart from the change in the order of the terms inside the absolute value in Eq. (65), no other action is necessary, and the integration over $y$ is performed as before. In the second case, however, care must be taken when the inflection point at $y=y_{0}$ is reached. Then, we write

$$
\begin{aligned}
\Phi_{ \pm}(y)= & \pm k \sqrt{\frac{2 f_{0} \mu^{2}}{45}} \\
& \times\left[\int_{0}^{y_{0}} d y^{\prime} \exp \left(-\frac{\mu^{2}}{2} e^{\frac{2 k y^{\prime}}{3}}+\frac{k y^{\prime}}{3}\right)\left(5-\mu^{2} e^{\frac{2 k k^{\prime}}{3}}\right)\right. \\
& \left.+\int_{y_{0}}^{y} d y^{\prime} \exp \left(-\frac{\mu^{2}}{2} e^{\frac{2 k y^{\prime}}{3}}+\frac{k y^{\prime}}{3}\right)\left(\mu^{2} e^{\frac{2 k y^{\prime}}{3}}-5\right)\right] .
\end{aligned}
$$

Overall, for $\mu^{2}<5$, the solution for the scalar field is

$\Phi_{ \pm}(y)=\left\{\begin{array}{cc}\mp[\mathcal{I}(y)-\mathcal{I}(0)], & y \leq y_{0}, \\ \pm\left[\mathcal{I}(0)-2 \mathcal{I}\left(y_{0}\right)+\mathcal{I}(y)\right], & y>y_{0},\end{array}\right\}$

where $\mathcal{I}(y)$ is still given by Eq. (67).

In Fig. 3(a), we depict the form of the warp factor $e^{-2 k|y|}$ and the coupling function $f(y)=f_{0} e^{-\mu^{2} e^{2 k y / 3}}$ in terms of the coordinate $y$ along the fifth dimension, for $f_{0}=500$, $k=1$, and $\mu^{2}=7$. Both functions exhibit a localization close to the brane with the coupling function $f(y)$ decreasing, in fact, much faster due to its double exponential dependence on $y$. At the boundary of space-time, both functions go smoothly to 0 . The displayed, qualitative behavior of these two quantities is independent of the particular values of the parameters. In contrast, the profile of the scalar field $\Phi(y)$ with respect to the extra dimension $y$ depends strongly on the value of the parameter $\mu^{2}$, as one may clearly see in Fig. 3(b). We observe that the behavior of the scalar field changes significantly as the parameter $\mu^{2}$ approaches and then surpasses the value 5. Indeed, for $\mu^{2}<5$, the emergence of the inflection point at $y=y_{0}>0$ is clearly visible. As $\mu^{2}$ approaches the value 5, the 


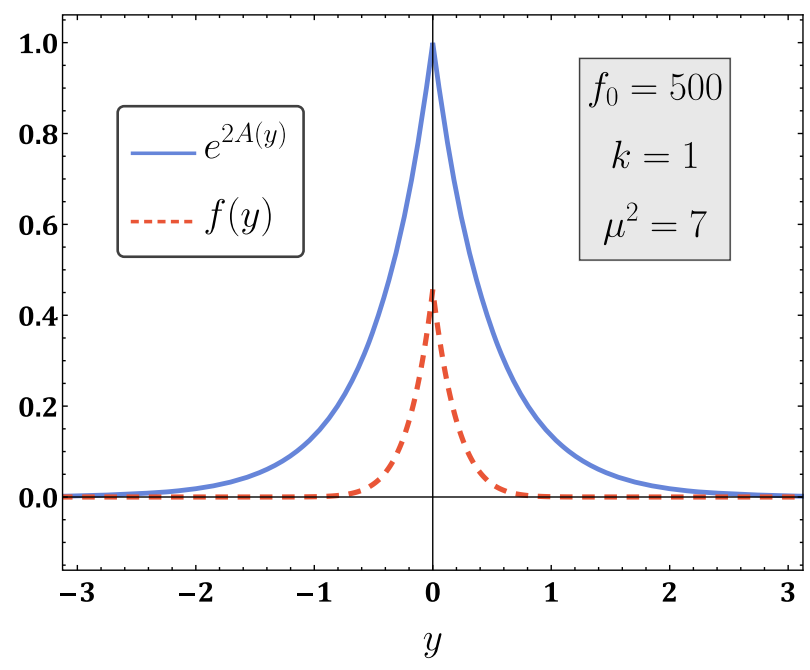

(a)

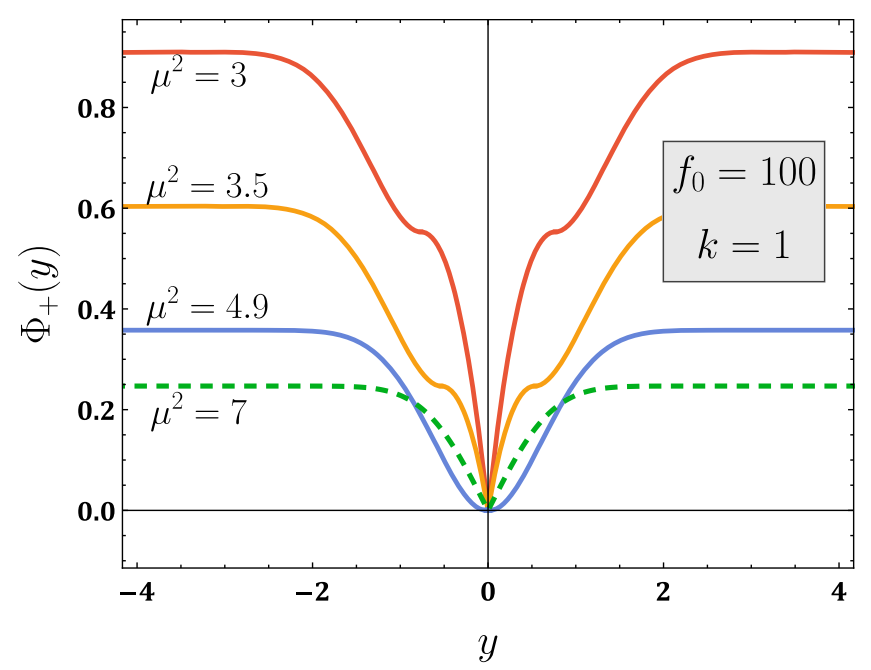

(b)

FIG. 3. (a) The warp factor $e^{2 A(y)}=e^{-2 k|y|}$ and the coupling function $f(y)=f_{0} e^{-\mu^{2} e^{2 k y / 3}}$, and (b) the scalar field $\Phi_{+}(y)$ for various values of the parameter $\mu^{2}$, in terms of the coordinate $y$.

inflection point moves towards the brane. For $\mu^{2} \geq 5$, though, this feature completely disappears in accordance with the analytical study presented above. Overall, the scalar field exhibits a monotonic behavior over the entire bulk-for the $\Phi_{+}(y)$ solution that we have chosen here to plot, the scalar field presents an increasing profile in the bulk reaching a constant, asymptotic value at the boundary of space-time. In Figs. 4(a) and 4(b), we present the dependence of $\Phi_{+}(y)$ on the second parameter $k$, since the parameter $\lambda$ is now fixed to the value of the warping parameter $k$ through the first of Eqs. (62); henceforth, we drop any reference to $\lambda$. We observe again the emergence of the inflection point when $\mu^{2}<5$, in Fig. 4(a), and the smooth behavior when $\mu^{2}>5$, in Fig. 4(b). The value of the warping parameter $k$ causes only a rise in the slope of the curve, as $k$ increases, leaving all the other features invariant.

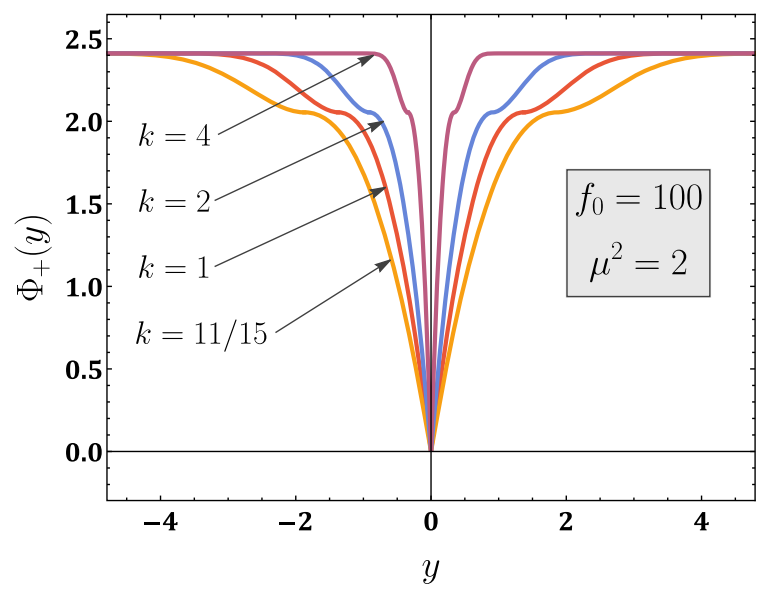

(a)
The bulk potential of the field $V_{B}$ in this case can be determined again by the general expression (43). Substituting the double exponential form of the coupling function $f(y)$, we now obtain the result

$$
\begin{aligned}
V_{B}(y)= & -\Lambda_{5}-f_{0} e^{-\mu^{2} e^{\frac{2 k y}{3}}} \\
& \times\left[6 k^{2}+\frac{k^{2} \mu^{2}}{45} e^{\frac{2 k y}{3}}\left(3 \mu^{4} e^{\frac{4 k y}{3}}+10 \mu^{2} e^{\frac{2 k y}{3}}+95\right)\right] .
\end{aligned}
$$

As in the simple exponential case, the combination inside the square brackets in the above expression is positive definite, for all values of the parameters of the model, thus rendering the second term of the bulk potential negative definite. Therefore, the presence of a nonminimally coupled scalar field in the bulk leads to a negative

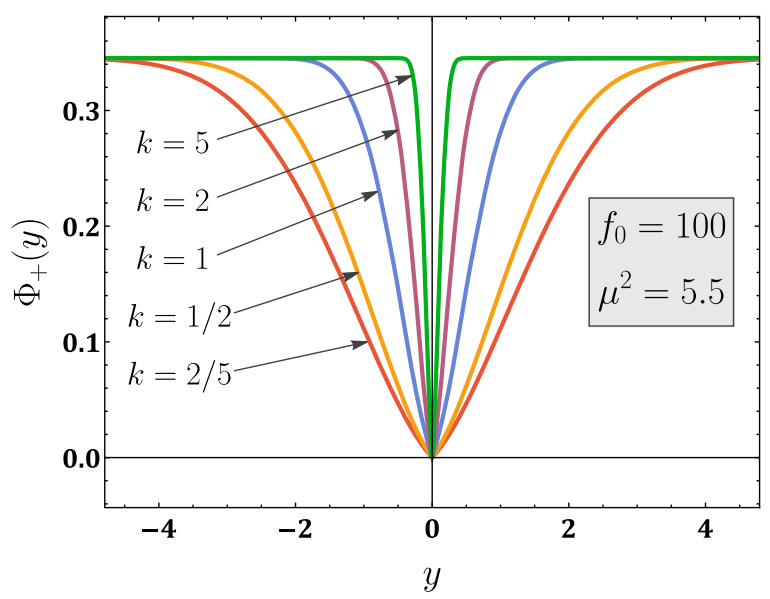

(b)

FIG. 4. The scalar field $\Phi_{+}(y)$, in terms of the coordinate $y$, for $f_{0}=100$ and for (a) $\mu^{2}=2$, and (b) $\mu^{2}=5.5$ and various values of $k$. 


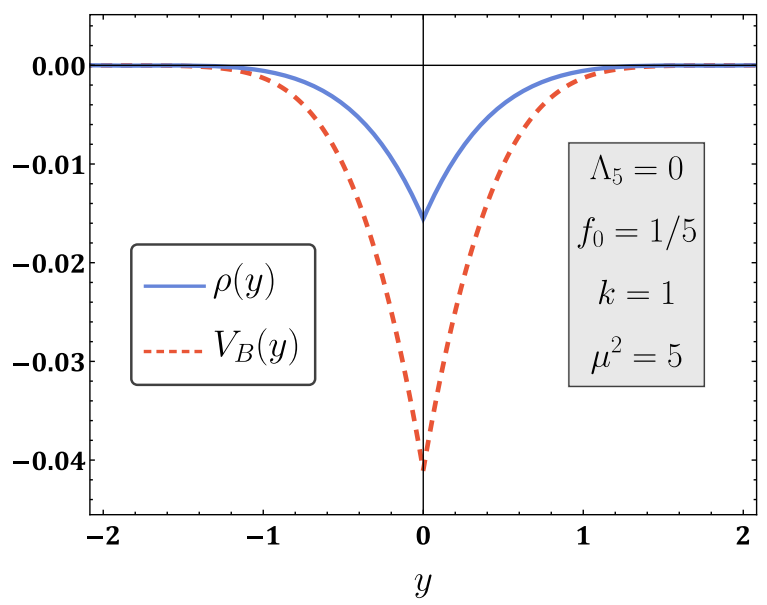

(a)

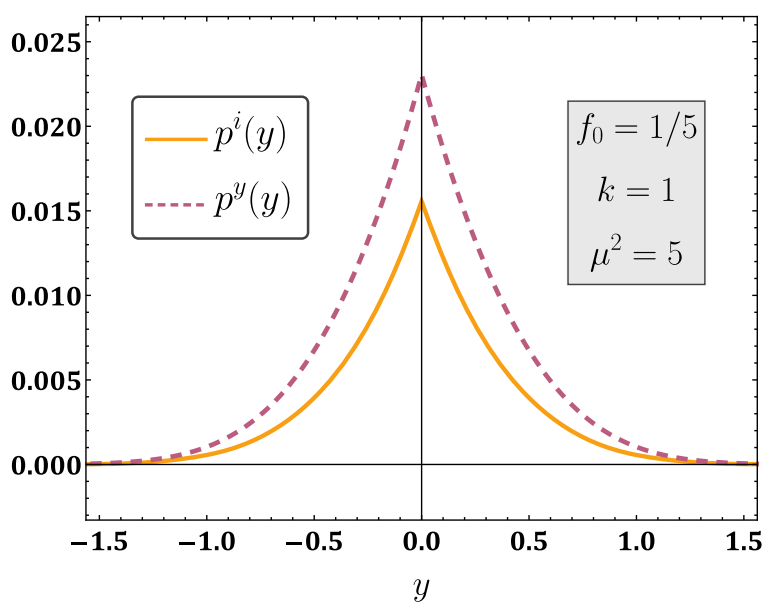

(b)

FIG. 5. (a) The scalar potential $V_{B}$ and energy density $\rho$ of the system, and (b) the pressure components $p^{y}$ and $p^{i}$ in terms of the coordinate $y$.

(nonconstant) potential energy in the bulk that can support again an AdS-type bulk space-time with an exponentially decreasing warp factor, even if the quantity $\Lambda_{5}$ is set to 0 . The potential has a smooth form over the entire bulk, is localized close to the brane, and goes to 0 extremely fast away from it-all these features are inherited from the form of the coupling function to which $V_{B}$ is directly proportional as Eq. (71) clearly shows. The aforementioned behavior of $V_{B}$ is depicted in Fig. 5(a) for $f_{0}=1 / 5$, $k=1$, and $\mu^{2}=5$.

The components of the energy-momentum tensor of the theory may be computed employing again Eqs. (45) and (46). Substituting again the form of the coupling function together with the expression for the bulk potential (71) presented above, we find the explicit expressions

$$
\begin{gathered}
\rho=-p^{i}=-2 f_{0} k^{2}\left(3+\frac{\mu^{6}}{45} e^{2 k y}\right) e^{-\mu^{2} e^{\frac{2 k y}{3}}}, \\
p^{y}=2 f_{0} k^{2}\left(3+\frac{2 \mu^{6}}{45} e^{2 k y}\right) e^{-\mu^{2} e^{\frac{2 k y}{3}}} .
\end{gathered}
$$

In Figs. 5(a) and 5(b) we present the behavior of the energy density $\rho$ and the pressure components $p^{i}$ and $p^{y}$, respectively, in terms of the extra dimension $y$. These quantities, too, present a smooth profile over the entire bulk, remain localized close to our brane, and vanish asymptotically, leaving behind a five-dimensional, flat space-time (if $\Lambda_{5}$ is assumed 0 ). The energy density $\rho$ is again negative throughout the bulk (as it should be in order to support by itself a pseudo-AdS space-time), but this is due to the presence of a physical, scalar d.o.f. coupled nonminimally to gravity with a physically acceptable positive-definite, and localized-close-to-our brane, coupling function.

\section{B. Junction conditions and effective theory}

For the junction conditions, we use again the general expressions (49) and (50), in which we substitute the form of the coupling function and employ also Eq. (65) to replace $\Phi^{\prime}(y)$. Then, Eq. (50) straightforwardly leads to the constraint

$$
2 k f_{0} e^{-\mu^{2}}\left(3+\frac{2 \mu^{2}}{3}\right)=\sigma+\left.V_{b}(\Phi)\right|_{y=0}
$$

which may be used again to fix the warping parameter $k$ in terms of the parameters $f_{0}$ and $\mu$ of the coupling function and the energy content $\left(\sigma+V_{b}\right)$ of the brane. On the other hand, Eq. (49) results $^{6}$ in the condition

$$
V_{b}^{\prime}(0)=f_{0} e^{-\mu^{2}} \frac{4 \mu^{2} k^{2}}{3}\left[\frac{\left(\mu^{2}-5\right)^{2}}{15}-4\right] .
$$

For a nontrivial $V_{b}$, the above condition can be used to restrict a parameter that may appear in its expression; on the other hand, for a trivial $V_{b}$, setting $V_{b}^{\prime}(0)=0$ in Eq. (75), we may fix also the value of $\mu^{2}$, and, through Eq. (62), the value of the effective cosmological constant on the brane $\Lambda=-\tilde{\Lambda}^{2}$.

We turn finally to the four-dimensional, effective theory on the brane. In order to derive the effective gravitational constant on the brane, we may use again the relation (54). Substituting the double exponential form of the coupling function $f(y)$, we find

\footnotetext{
${ }^{6}$ Note that care should be taken in the evaluation of (49) due to the different behavior of the scalar field $\Phi(y)$ in terms of $\mu^{2}$. At the end of the evaluation, though, a unique expression follows from this junction condition for either $\mu^{2}>5$ or $\mu^{2}<5$.
} 


$$
\begin{aligned}
\frac{1}{\kappa_{4}^{2}} & =2 f_{0} \int_{0}^{\infty} d y e^{-2 k y} \exp \left(-\mu^{2} e^{\frac{2 k y}{3}}\right) \\
& =\frac{f_{0}}{2 k}\left[e^{-\mu^{2}}\left(2-\mu^{2}+\mu^{4}\right)+\mu^{6} \operatorname{Ei}\left(-\mu^{2}\right)\right] .
\end{aligned}
$$

Above, we have used the exponential-integral function $\operatorname{Ei}(x)$ defined as

$$
\operatorname{Ei}(x) \equiv-\int_{-x}^{\infty} d t \frac{e^{-t}}{t}
$$

and its property that $\lim _{x \rightarrow \infty} \operatorname{Ei}(-x)=0$. For $x<0$, it can also be shown that [92]

$$
\operatorname{Ei}(x)=\operatorname{Ei}(-|x|)=\gamma+\ln |x|+\sum_{k=1}^{\infty} \frac{(-1)^{k}|x|^{k}}{k k !},
$$

where $\gamma$ is the Euler-Mascheroni constant. Then, the effective gravitational energy scale is given by

$$
\begin{aligned}
M_{\mathrm{Pl}}^{2}= & \frac{4 \pi f_{0}}{k}\left\{e^{-\mu^{2}}\left(2-\mu^{2}+\mu^{4}\right)\right. \\
& \left.+\mu^{6}\left[\gamma+2 \ln |\mu|+\sum_{q=1}^{\infty} \frac{(-1)^{q} \mu^{2 q}}{q q !}\right]\right\} .
\end{aligned}
$$

Once again, the gravity scale on the brane $M_{P l^{2}}$ is determined primarily by the ratio $f_{0} / k \sim M_{*}^{3} / k$, which resembles again the corresponding relation of the RandallSundrum model [12]-note that the combination inside the curly brackets in Eq. (76) is of $\mathcal{O}(1)$.

The effective cosmological constant on the brane can be evaluated by employing Eq. (57). Using again the form of the coupling function $f(y)$ and the junction condition (74), we find that

$$
\begin{aligned}
\Lambda_{4} & =-\frac{f_{0} k \mu^{6}}{45}\left[e^{-\mu^{2}}\left(2-\mu^{2}+\mu^{4}\right)+\mu^{6} \operatorname{Ei}\left(-\mu^{2}\right)\right] \\
& =-\frac{\tilde{\Lambda}^{2}}{\kappa_{4}^{2}}=\frac{\Lambda}{\kappa_{4}^{2}} .
\end{aligned}
$$

Above, we have used the second of Eqs. (62), which relates the parameter $\mu^{2}$ with $\tilde{\Lambda}^{2}$, and the expression (76) for $\kappa_{4}^{2}$. As in the model discussed in Sec. III, the integration parameter $\Lambda$ is confirmed to be the effective cosmological constant $\Lambda_{4}$ on the brane multiplied by the effective gravitational constant $\kappa_{4}^{2}$.

\section{CONCLUSIONS}

In this work, we have considered a five-dimensional gravitational theory containing a scalar field with a nonminimal coupling to the five-dimensional Ricci scalar. The coupling is realized through a smooth, real, positive-definite coupling function $f(\Phi)$. Demanding that all components of the energy-momentum tensor remain finite throughout the bulk, and looking for analytic solutions for the scalar field, we have restricted our choices for the coupling function to two particular forms: a simple exponential and a double exponential, both decreasing away from the brane. This results in a scalar-tensor fivedimensional theory with a nonminimal coupling between the scalar field and gravity that is effectively localized close to the brane.

The five-dimensional line element contained two, initially arbitrary, functions: the warp factor and the mass function. The warp factor was chosen to have the RandallSundrum form in order to ensure the gravity localization close to our brane. The mass function was uniquely determined by integrating the gravitational field equations and turned out to have a form that, on the brane, produces a Schwarzschild-(anti-) de Sitter space-time. Having studied the case of a positive effective cosmological constant on the brane in a previous work of ours [74], here, we focused on the case of a negative four-dimensional cosmological constant. In fact, for this choice, the nonminimal coupling function is allowed to have everywhere a positive value and thus to guarantee a normal gravitational force both in the bulk and on the brane.

The solution for the mass function contained also a mass parameter that was identified with the mass of the black hole that a four-dimensional observer sees on the brane. However, the calculation of the five-dimensional scalarinvariant quantities revealed that, for a nonvanishing mass parameter, the five-dimensional solution is in fact a black string plagued by a singularity extending over the entire extra dimension. It is again remarkable how easily the classical, ill-defined black-string solutions emerge in the context of physically plausible scalar-field theories, whereas classical black-hole solutions are still eluding us. If we set this mass parameter equal to 0 , then the extended singularity disappears leaving behind a maximally symmetric space-time on the brane and a regular fivedimensional bulk with a true singularity at the boundary of space-time.

In order to complete the solution, the form of the scalar field in the bulk also had to be determined. We have managed to analytically attack the problem of the integration of the scalar-field equation and to derive two particular solutions. For the exponentially decreasing coupling function, the scalar field was expressed in terms of the hypergeometric function (which, for particular values of the parameters, was further reduced to a combination of elementary functions) while for the double exponential form of $f(y)$, the scalar field was given by a combination of an exponential and the error function. In both cases, the profile of the scalar field presented the same set of robust features: $\Phi(y)$ was everywhere a smooth, regular, monotonic function of the extra coordinate approaching a constant, finite value at the boundaries of space-time. 
The same robust behavior was exhibited also by the bulk potential of the scalar field and all components of the energy-momentum tensor. All the aforementioned quantities were finite everywhere in the bulk, remained localized close to our brane, and vanished asymptotically at large distances. In particular, the scalar potential was everywhere negative definite, which led also to a negative-definite energy density. However, this negative distribution of energy was generated, not by an exotic form of matter, but by a physical, scalar d.o.f. coupled nonminimally to gravity with a positive-definite, and localized-close-to-ourbrane, coupling function. This energy may therefore support by itself a pseudo-AdS space-time, even in the absence of a negative, bulk cosmological constant, and thus ensure the localization of gravity close to our brane.

The presence of the brane in the theory introduces a set of junction conditions that may serve to fix two of the parameters of the theory, preferably the warp-factor parameter $k$ and a parameter of the interaction term of the scalar field with the brane. If the latter term is nontrivial, the effective cosmological constant remains a free parameter of the model; however, for a trivial scalar-brane interaction term, the value of the cosmological constant on the brane may be uniquely determined. The calculation of the effective theory on the brane led to a finite theory, as expected, without the need to introduce a second brane in the model. The relation between the fundamental and the effective gravitational scale had a similar form with the one emerging in the Randall-Sundrum model, although our theory has a dynamical, more realistic field content.

In order to produce finite, analytic solutions for the bulk scalar field, we have made indeed two particular choices for the nonminimal coupling function. However, despite the apparently different explicit forms of the scalar field, the main characteristics of the two solutions remained the same, namely, the smoothness, the regularity, even the constant, finite value at infinity. This type of "universality" is caused by the common characteristics that the corresponding forms of the coupling function had: they were both smooth, well defined throughout the bulk, positive definite, and localized close to our brane. We may therefore assert that the two particular solutions we have derived are in fact representative of the behavior that a generic solution for the scalar field would exhibit if it was sourced by the Ricci scalar through any coupling function that would respect the same finiteness and positivity-of-value criteria.

Let us stress again that the solutions presented in this work are supported by a bulk distribution of energy related to a physically acceptable, realistic scalar-tensor theory of gravity. Both of our solutions are characterized by an exponentially decreasing warp factor, identical to that of Refs. [11,12], without assuming that the sole energy content of the theory in the bulk is a negative cosmological constant-rather, it is a nontrivial scalar field with a localized, negative potential that supports this feature.
Our solutions reduce to a black-hole line element on the brane, as in Ref. [16], if we assume that $M \neq 0$, but allow also for the presence of a nonzero cosmological constant on the brane. This feature was absent in the analysis of [16] where the brane was assumed to be flat. Allowing for a nonzero cosmological constant on the brane has also revealed that the bulk possesses then an additional singularity located at an infinite coordinate distance from our brane in the case where $M=0$. This singularity does not affect either the profile of the scalar field in the bulk or the effective theory on the brane, but, if desired, it could easily be shielded by the introduction of a second brane.

Finally, the stability behavior of our solutions is an important aspect that needs to be studied. Compared to the existing stability analyses performed along the lines of Refs. [59,60], our theory has the additional complexity of the presence of the scalar field. Our solutions are not purely gravitational; therefore, the perturbation analysis involves a coupled system of scalar-field and gravitational equations. The sign of the cosmological constant $\Lambda$ and the corresponding properties of the nonminimal coupling function $f(\Phi)$ of the scalar field to the Ricci scalar are also expected to play a role in this analysis. Such an analysis will reveal whether the scalar field may stabilize the black string over the bulk regime close to our brane where it has a nontrivial profile. However, beyond the point where the scalar-field energy-momentum tensor vanishes, we expect the GregoryLaflamme instability to set in, as in all other infinitely extended black-string solutions. The stability of the solutions for the case of $M=0$ should also be carefully examined as the role of the singularity, arising at the boundary of space-time when $\Lambda \neq 0$, may be found to be important. We hope to return to these questions soon.

\section{ACKNOWLEDGMENTS}

The research of T. N. was cofinanced by Greece and the European Union [European Social Fund (ESF)] through the operational program "Human resources development, education, and lifelong learning" in the context of the project "Strengthening human resources research potential via doctorate research" (Grant No. MIS-5000432), implemented by the State Scholarships Foundation (IKY). The research of N.P. was implemented under the "Strengthening postdoctoral research" scholarship program (Grant No. 2016-050-0503-7626) by the Hellenic State Scholarship Foundation as part of the operational program "Human resources development, education, and lifelong learning," cofinanced by the ESF and the Greek government.

\section{APPENDIX A: CURVATURE INVARIANT QUANTITIES}

Employing the form of the five-dimensional line element (6), we compute the following scalar invariant quantities: 


$$
\begin{gathered}
R=-8 A^{\prime \prime}-20 A^{\prime 2}+\frac{2 e^{-2 A}\left(r \partial_{r}^{2} m+2 \partial_{r} m\right)}{r^{2}} \\
R_{M N} R^{M N}=2 e^{-4 A}\left[e^{2 A}\left(A^{\prime \prime}+4 A^{\prime 2}\right)-\frac{\partial_{r}^{2} m}{r}\right]^{2}+2 \frac{e^{-4 A}\left[r^{2} e^{2 A}\left(A^{\prime \prime}+4 A^{\prime 2}\right)-2 \partial_{r} m\right]^{2}}{r^{4}} \\
+16\left(A^{\prime \prime}+A^{\prime 2}\right)^{2}, \\
R_{M N K L} R^{M N K L}=-\frac{8 e^{-2 A} A^{\prime 2}\left(r \partial_{r}^{2} m+2 \partial_{r} m\right)}{r^{2}}+40 A^{\prime 4}+16 A^{\prime \prime}\left(A^{\prime \prime}+2 A^{\prime 2}\right) \\
+4 e^{-4 A}\left[\frac{\left(\partial_{r}^{2} m\right)^{2}}{r^{2}}+\frac{4\left[2\left(\partial_{r} m\right)^{2}+\left(m-r \partial_{r} m\right) \partial_{r}^{2} m\right]}{r^{4}}+\frac{4\left(3 m^{2}-4 r m \partial_{r} m\right)}{r^{6}}\right] .
\end{gathered}
$$

\section{APPENDIX B: A SYSTEMATIC METHODOLOGY TO EXPRESS ${ }_{2} F_{1}\left(\frac{3}{2}-\frac{\lambda}{4}, \frac{3}{2} ; \frac{5}{2} ; u^{2}\right)$ IN TERMS OF ELEMENTARY FUNCTIONS WHEN $\lambda=4 q$, AND $q \in \mathbb{Z}^{>}$}

Let us start with the simplest case of $\lambda=4$, i.e., $q=1$. For simplicity, we use the variable $u^{2}=\frac{w-1}{w}$. Then, using the expansion of the hypergeometric function given in Eq. (36) and setting $\lambda=4$, we readily obtain

$$
{ }_{2} F_{1}\left(\frac{1}{2}, \frac{3}{2} ; \frac{5}{2} ; u^{2}\right)=\frac{3}{2 \sqrt{\pi}} \sum_{n=0}^{\infty} \frac{\Gamma\left(n+\frac{1}{2}\right)}{n+\frac{3}{2}} \frac{u^{2 n}}{n !}=\frac{3}{2 \sqrt{\pi}} \sum_{n=0}^{\infty} \frac{2 n-1}{2 n+3} \Gamma\left(n-\frac{1}{2}\right) \frac{u^{2 n}}{n !},
$$

where we have used the gamma function property $\Gamma(1+z)=z \Gamma(z)$. In order to express the above in terms of elementary functions, we observe the following:

$$
\begin{aligned}
\frac{\arcsin u}{u}-\sqrt{1-u^{2}} & ={ }_{2} F_{1}\left(\frac{1}{2}, \frac{1}{2} ; \frac{3}{2} ; u^{2}\right)-{ }_{2} F_{1}\left(-\frac{1}{2}, 1 ; 1 ; u^{2}\right) \\
& =\sum_{n=0}^{\infty}\left[\frac{\Gamma\left(n+\frac{1}{2}\right)}{(2 n+1) \sqrt{\pi}}+\frac{\Gamma\left(n-\frac{1}{2}\right)}{2 \sqrt{\pi}}\right] \frac{u^{2 n}}{n !}=\sum_{n=1}^{\infty} \frac{2 n}{2 n+1} \frac{\Gamma\left(n-\frac{1}{2}\right)}{\sqrt{\pi}} \frac{u^{2 n}}{n !} \\
& =\sum_{m=0}^{\infty} \frac{2(m+1)}{2 m+3} \frac{\Gamma\left(m+\frac{1}{2}\right)}{\sqrt{\pi}} \frac{u^{2(m+1)}}{(m+1) !}=\frac{1}{\sqrt{\pi}} \sum_{m=0}^{\infty} \frac{2 m-1}{2 m+3} \Gamma\left(m-\frac{1}{2}\right) \frac{u^{2(m+1)}}{m !} .
\end{aligned}
$$

Note that, in the second sum of the second line of the above equation, we have changed the lower value of $n$ from $n=0$ to $n=1$ since, due to the ( $2 n)$ factor, this value has a trivial contribution to the sum. Subsequently, we set $n=m+1$, and by rearranging we arrived at the final result. Comparing now Eqs. (B1) and (B2), we find that

$$
{ }_{2} F_{1}\left(\frac{1}{2}, \frac{3}{2} ; \frac{5}{2} ; u^{2}\right) u^{2}=\frac{3}{2}\left(\frac{\arcsin u}{u}-\sqrt{1-u^{2}}\right)
$$

Let us now address the more general case where $\lambda=4 q$, with $q=1+\ell$, where $\ell$ a positive number. Then, applying again Eq. (36), we may write

$$
{ }_{2} F_{1}\left(\frac{1}{2}-\ell, \frac{3}{2} ; \frac{5}{2} ; u^{2}\right)=\frac{3}{\sqrt{\pi}} \frac{(2 \ell) !}{\ell !(-4)^{\ell}} \sum_{n=0}^{\infty} \frac{1}{2 n+3} \Gamma\left(n-\ell+\frac{1}{2}\right) \frac{u^{2 n}}{n !},
$$

where we have also used the property $\Gamma\left(-\ell+\frac{1}{2}\right)=\frac{\ell !(-4)^{\ell} \sqrt{\pi}}{(2 \ell) !}$. But, it also holds that

$$
\begin{aligned}
\Gamma\left(n-\frac{1}{2}\right)=\frac{\Gamma\left(n-\ell+\frac{1}{2}+\ell\right)}{n-\frac{1}{2}} & =\frac{2}{2 n-1}\left(n-\frac{1}{2}\right)\left(n-\frac{3}{2}\right) \cdots\left(n-\ell+\frac{1}{2}\right) \Gamma\left(n-\ell+\frac{1}{2}\right) \\
& =2^{1-\ell} \frac{(2 n-1)(2 n-3) \cdots(2 n-2 \ell+1)}{2 n-1} \Gamma\left(n-\ell+\frac{1}{2}\right) .
\end{aligned}
$$


From Eqs. (B2) and (B5), we then obtain

$$
\frac{\arcsin (u)}{u}-\sqrt{1-u^{2}}=\frac{2^{1-\ell}}{\sqrt{\pi}} \sum_{n=0}^{\infty} \frac{(2 n-1)(2 n-3) \cdots(2 n-2 \ell+1)}{2 n+3} \Gamma\left(n-\ell+\frac{1}{2}\right) \frac{u^{2(n+1)}}{n !} .
$$

In what follows, we discuss also how the multiplication between even powers of $u$ and $\sqrt{1-u^{2}}$ can result in similar expansions as the one in Eq. (B6). The obtained expansions together with Eq. (B6) help us to express the rhs of Eq. (B4) in terms of elementary functions. Thus, starting from the relation

$$
\sqrt{1-u^{2}}=-\frac{1}{2 \sqrt{\pi}} \sum_{n=0}^{\infty} \Gamma\left(n-\frac{1}{2}\right) \frac{u^{2 n}}{n !}
$$

we write, employing also Eq. (B5),

$$
u^{2} \sqrt{1-u^{2}}=-\frac{2^{1-\ell}}{2 \sqrt{\pi}} \sum_{n=0}^{\infty} \frac{(2 n-1)(2 n-3) \cdots(2 n-2 \ell+1)}{2 n-1} \Gamma\left(n-\ell+\frac{1}{2}\right) \frac{u^{2(n+1)}}{n !} .
$$

Similarly, we obtain

$$
\begin{aligned}
u^{4} \sqrt{1-u^{2}} & =-\frac{1}{2 \sqrt{\pi}} \sum_{m=0}^{\infty} m \Gamma\left(m-\frac{3}{2}\right) \frac{u^{2(m+1)}}{m !}=-\frac{1}{2 \sqrt{\pi}} \sum_{m=0}^{\infty} \frac{2 m}{2 m-3} \Gamma\left(m-\frac{1}{2}\right) \frac{u^{2(m+1)}}{m !} \\
& =-\frac{2^{2-\ell}}{2 \sqrt{\pi}} \sum_{n=0}^{\infty} \frac{(2 n-1)(2 n-3) \cdots(2 n-2 \ell+1) n}{(2 n-1)(2 n-3)} \Gamma\left(n-\ell+\frac{1}{2}\right) \frac{u^{2(n+1)}}{n !}
\end{aligned}
$$

Note that, in the first sum of the above expression, we set $m=n+1$ but retained the lower value of the sum to be 0 due to the $m$ factor-we have also used, once again, Eq. (B5). Continuing along the same lines, we obtain, for a general $\ell$, the result

$$
\begin{aligned}
u^{2 \ell} \sqrt{1-u^{2}} & =-\frac{1}{2 \sqrt{\pi}} \sum_{m=\ell-1}^{\infty} m(m-1) \cdots(m-\ell+2) \Gamma\left(m-\ell+\frac{1}{2}\right) \frac{u^{2(m+1)}}{m !} \\
& =-\frac{1}{2 \sqrt{\pi}} \sum_{n=0}^{\infty} n(n-1) \cdots(n-\ell+2) \Gamma\left(n-\ell+\frac{1}{2}\right) \frac{u^{2(n+1)}}{n !},
\end{aligned}
$$

where, now, we set $m=n+\ell-1$ and again reinstated the lower value of the sum to be 0 due to the multiplying factors that trivialize all terms with $n<\ell-1$.

Comparing now the rhs's of Eqs. (B6), (B8), and (B10) with the rhs of Eq. (B4), we conclude that we may express the aforementioned hypergeometric function as

$$
\begin{aligned}
& { }_{2} F_{1}\left(\frac{1}{2}-\ell, \frac{3}{2} ; \frac{5}{2} ; u^{2}\right) u^{2}=\alpha\left(\frac{\arcsin u}{u}-\sqrt{1-u^{2}}\right) \\
& \quad+\sqrt{1-u^{2}}\left(\beta_{1} u^{2}+\beta_{2} u^{4}+\cdots+\beta_{\ell-1} u^{2(\ell-1)}+\beta_{\ell} u^{2 \ell}\right),
\end{aligned}
$$

where $\alpha, \beta_{1}, \ldots, \beta_{\ell}$ are constant coefficients. These may be determined by substituting the explicit expansions (B4), (B6), (B8), and (B10) on both sides of the above equation and demanding its validity. Then, we obtain the following relation for the coefficients $\alpha, \beta_{1}, \ldots, \beta_{\ell}$, which must be true for arbitrary $n \in \mathbb{Z}^{\geq}$, 


$$
\begin{aligned}
6 \frac{(2 \ell) !}{\ell !(-4)^{\ell}}= & \alpha 2^{2-\ell}(2 n-1)(2 n-3) \cdots(2 n-2 \ell+1) \\
& -(2 n+3)\left[\beta_{1} 2^{1-\ell} \frac{(2 n-1)(2 n-3) \cdots(2 n-2 \ell+1)}{2 n-1}\right. \\
& +\beta_{2} 2^{2-\ell} \frac{(2 n-1)(2 n-3) \cdots(2 n-2 \ell+1)}{(2 n-1)(2 n-3)} n \\
& +\beta_{3} 2^{3-\ell} \frac{(2 n-1)(2 n-3) \cdots(2 n-2 \ell+1)}{(2 n-1)(2 n-3)(2 n-5)} n(n-1) \\
& \vdots \\
& +\beta_{\ell-1} 2^{-1}(2 n-2 \ell+1) n(n-1) \cdots(n-\ell+3) \\
& \left.+\beta_{\ell} \frac{(n+1) n(n-1) \cdots(n-\ell+2)}{n+1}\right] .
\end{aligned}
$$

The above equation leads to a system of $\ell+1$ linear equations with $\ell+1$ variables from which the unknown coefficients $\alpha, \beta_{1}, \ldots, \beta_{\ell}$ may easily be derived.

[1] A. Einstein, Zur allgemeinen Relativitätstheorie, Sitzungsberichte der Königlich Preußischen Akademie der Wissenschaften, 1915, https://ui.adsabs.harvard.edu/abs/ 1915SPAW.......778E.

[2] A. Einstein, Die Feldgleichungen der Gravitation, Sitzungsberichte der Königlich Preußischen Akademie der Wissenschaften, 1915, https://ui.adsabs.harvard.edu/abs/ 1915SPAW.......844E.

[3] A. Einstein, Die Grundlage der allgemeinen RelativitätstheorieDie Grundlage der allgemeinen Relativitätstheorie, Ann. Phys. (Berlin) 354, 769 (1916).

[4] Th. Kaluza, On the unification problem in physics, Int. J. Mod. Phys. D 27, 1870001 (2018).

[5] O. Klein, Quantum theory and five-dimensional theory of relativity, Z. Phys. 37, 895 (1926).

[6] V. A. Rubakov and M. E. Shaposhnikov, Do we live inside a domain wall?, Phys. Lett. B 125, 136 (1983).

[7] K. Akama, An early proposal of "Brane World", Lect. Notes Phys. 176, 267 (1982).

[8] N. Arkani-Hamed, S. Dimopoulos, and G. Dvali, The hierarchy problem and new dimensions at a millimeter, Phys. Lett. B 429, 263 (1998).

[9] N. Arkani-Hamed, S. Dimopoulos, and G. Dvali, Phenomenology, astrophysics, and cosmology of theories with submillimeter dimensions and $\mathrm{TeV}$ scale quantum gravity, Phys. Rev. D 59, 086004 (1999).

[10] I. Antoniadis, N. Arkani-Hamed, S. Dimopoulos, and G. Dvali, New dimensions at a millimeter to a fermi and superstrings at a TeV, Phys. Lett. B 436, 257 (1998).

[11] L. Randall and R. Sundrum, Large Mass Hierarchy from a Small Extra Dimension, Phys. Rev. Lett. 83, 3370 (1999).

[12] L. Randall and R. Sundrum, An Alternative to Compactification, Phys. Rev. Lett. 83, 4690 (1999).
[13] C. W. Misner, K. S. Thorne, and J. A. Wheeler, Gravitation (W. H. Freeman, San Francisco, 1973).

[14] B. Carter, Axisymmetric Black Hole Has Only Two Degrees of Freedom, Phys. Rev. Lett. 26, 331 (1971).

[15] R. Emparan and H. S. Reall, Black holes in higher dimensions, Living Rev. Relativity 11, 6 (2008).

[16] A. Chamblin, S. W. Hawking, and H. S. Reall, Brane-world black holes, Phys. Rev. D 61, 065007 (2000).

[17] R. Emparan, G. T. Horowitz, and R. C. Myers, Exact description of black holes on branes, J. High Energy Phys. 01 (2000) 007.

[18] R. Emparan, G. T. Horowitz, and R. C. Myers, Exact description of black holes on branes II: comparison with BTZ black holes and black strings, J. High Energy Phys. 01 (2000) 021.

[19] N. Dadhich, R. Maartens, P. Papadopoulos, and V. Rezania, Black holes on the brane, Phys. Lett. B 487, 1 (2000).

[20] P. Kanti and K. Tamvakis, Quest for localized 4D black holes in brane worlds, Phys. Rev. D 65, 084010 (2002).

[21] G. Kofinas, E. Papantonopoulos, and V. Zamarias, Black hole solutions in braneworlds with induced gravity, Phys. Rev. D 66, 104028 (2002).

[22] R. Casadio, A. Fabbri, and L. Mazzacurati, New black holes in the brane world?, Phys. Rev. D 65, 084040 (2002).

[23] V. Frolov, M. Snajdr, and D. Stojković, Interaction of a brane with a moving bulk black hole, Phys. Rev. D 68, 044002 (2003).

[24] P. Kanti, I. Olasagasti, and K. Tamvakis, Quest for localized 4D black holes in brane worlds. II. Removing the bulk singularities, Phys. Rev. D 68, 124001 (2003).

[25] G. Kofinas and E. Papantonopoulos, Gravitational collapse in braneworld models with curvature corrections, J. Cosmol. Astropart. Phys. 12 (2004) 011. 
[26] D. Karasik, C. Sahabandu, P. Suranyi, and L. C. R. Wijewardhana, Small black holes on branes: Is the horizon regular or singular?, Phys. Rev. D 70, 064007 (2004).

[27] C. Galfard, C. Germani, and A. Ishibashi, Asymptotically AdS brane black holes, Phys. Rev. D 73, 064014 (2006).

[28] S. Creek, R. Gregory, P. Kanti, and B. Mistry, Braneworld stars and black holes, Classical Quantum Gravity 23, 6633 (2006).

[29] B. Cuadros-Melgar, E. Papantonopoulos, M. Tsoukalas, and V. Zamarias, Bañados-Teitelboim-Zanellilike String on Codimension-2 Braneworlds in the Thin Brane Limit, Phys. Rev. Lett. 100, 221601 (2008).

[30] M. Anber and L. Sorbo, New exact solutions on the randallsundrum 2-brane: Lumps of dark radiation and accelerated black holes, J. High Energy Phys. 07 (2008) 098.

[31] M. Heydari-Fard and H. R. Sepangi, Spherically symmetric solutions and gravitational collapse in brane-worlds, J. Cosmol. Astropart. Phys. 02 (2009) 029.

[32] A. A. Andrianov and M. A. Kurkov, Black holes in the brane world: Some exact solutions, Theor. Math. Phys. 169, 1629 (2011).

[33] R. Casadio and J. Ovalle, Brane-world stars and (microscopic) black holes, Phys. Lett. B 715, 251 (2012).

[34] P. Kanti, N. Pappas, and K. Zuleta, On the localization of four-dimensional brane-world black holes, Classical Quantum Gravity 30, 235017 (2013).

[35] J. Ovalle and F. Linares, Tolman IV solution in the RandallSundrum braneworld, Phys. Rev. D 88, 104026 (2013).

[36] T. Harko and M. J. Lake, Null fluid collapse in brane world models, Phys. Rev. D 89, 064038 (2014).

[37] J. Ovalle, L. Á. Gergely, and R. Casadio, Brane-world stars with a solid crust and vacuum exterior, Classical Quantum Gravity 32, 045015 (2015).

[38] A. M. Kuerten and R. da Rocha, Probing topologically charged black holes on brane worlds in $f(R)$ bulk, Gen. Relativ. Gravit. 48, 90 (2016).

[39] A. Herrera-Aguilar, A. M. Kuerten, and R. da Rocha, Regular bulk solutions in Brane-worlds with inhomogeneous dust and generalized dark radiation, Adv. High Energy Phys. 2015, 1 (2015).

[40] R. Casadio, J. Ovalle, and R. da Rocha, The minimal geometric deformation approach extended, Classical Quantum Gravity 32, 215020 (2015).

[41] P. Kanti, N. Pappas, and T. Pappas, On the localization of four-dimensional brane-world black holes: II, The general case, Classical Quantum Gravity 33, 015003 (2016).

[42] R. Maartens and K. Koyama, Brane-World gravity, Living Rev. Relativity 13, 5 (2010).

[43] A. S. Majumdar and N. Mukherjee, Braneworld black holes in cosmology and astrophysics, Int. J. Mod. Phys. D 14, 1095 (2005).

[44] R. Gregory, Braneworld Black Holes (Springer, Berlin, Heidelberg, 2009), pp. 259-298.

[45] P. Kanti, Brane-world black holes, J. Phys. Conf. Ser. 189, 012020 (2009).

[46] P. Kanti, Footprints of higher-dimensional decaying black holes, Rom. J. Phys. 57, 879 (2012).

[47] P. Kanti and E. Winstanley, Hawking Radiation from Higher-Dimensional Black Holes (Springer International Publishing, Cham, 2015), p. 229.
[48] N. D. Pappas, The Black Hole Challenge in RandallSundrum II model (Nova Science Publishers, New York, 2014).

[49] T. Nakas, Searching for localized black-hole solutions in Brane-World models, Master's thesis, Ioannina University, 2017.

[50] H. Kudoh, T. Tanaka, and T. Nakamura, Small localized black holes in a braneworld: Formulation and numerical method, Phys. Rev. D 68, 024035 (2003).

[51] H. Kudoh, Six-dimensional localized black holes: Numerical solutions, Phys. Rev. D 69, 104019 (2004).

[52] N. Tanahashi and T. Tanaka, Time-symmetric initial data of large brane-localized black hole in RS-II model, J. High Energy Phys. 03 (2008) 041.

[53] B. Kleihaus, J. Kunz, E. Radu, and D. Senkbeil, Electric charge on the brane?, Phys. Rev. D 83, 104050 (2011).

[54] P. Figueras and T. Wiseman, Gravity and Large Black Holes in Randall-Sundrum II Braneworlds, Phys. Rev. Lett. 107, 081101 (2011).

[55] S. Abdolrahimi, C. Cattoën, D. N. Page, and S. YaghoobpourTari, Large Randall-Sundrum II black holes, Phys. Lett. B 720, 405 (2013).

[56] S. Abdolrahimi, C. Cattoën, D. N. Page, and S. YaghoobpourTari, Spectral methods in general relativity and large RandallSundrum II black holes, J. Cosmology Astropart. Phys. 06 (2013) 039.

[57] F. R. Tangherlini, Schwarzschild field in $\mathrm{n}$ dimensions and the dimensionality of space problem, Il Nuovo Cimento 27, 636 (1963).

[58] R. C. Myers and M.J. Perry, Black holes in higher dimensional space-times, Ann. Phys. (N.Y.) 172, 304 (1986).

[59] R. Gregory and R. Laflamme, Black Strings and p-Branes are Unstable, Phys. Rev. Lett. 70, 2837 (1993).

[60] R. Gregory, Black string instabilities in antide Sitter space, Classical Quantum Gravity 17, L125 (2000).

[61] S. S. Gubser, On nonuniform black branes, Classical Quantum Gravity 19, 4825 (2002).

[62] T. Wiseman, Static axisymmetric vacuum solutions and nonuniform black strings, Classical Quantum Gravity 20, 1137 (2003).

[63] H. Kudoh and T. Wiseman, Connecting Black Holes and Black Strings, Phys. Rev. Lett. 94, 161102 (2005).

[64] E. Sorkin, Critical Dimension in the Black-String Phase Transition, Phys. Rev. Lett. 93, 031601 (2004).

[65] E. Sorkin, Nonuniform black strings in various dimensions, Phys. Rev. D 74, 104027 (2006).

[66] B. Kleihaus, J. Kunz, and E. Radu, New nonuniform black string solutions, J. High Energy Phys. 06 (2006) 016.

[67] M. Headrick, S. Kitchen, and T. Wiseman, A new approach to static numerical relativity and its application to KaluzaKlein black holes, Classical Quantum Gravity 27, 035002 (2010).

[68] C. Bogdanos, C. Charmousis, B. Gouteraux, and R. Zegers, Einstein-Gauss-Bonnet metrics: Black holes, black strings and a staticity theorem, J. High Energy Phys. 10 (2009) 037.

[69] C. Charmousis, T. Kolyvaris, and E. Papantonopoulos, Charged C-metric with conformally coupled scalar field, Classical Quantum Gravity 26, 175012 (2009). 
[70] P. Figueras, K. Murata, and H. S. Reall, Stable nonuniform black strings below the critical dimension, J. High Energy Phys. 11 (2012) 071.

[71] M. Kalisch and M. Ansorg, Pseudo-spectral construction of nonuniform black string solutions in five and six space-time dimensions, Classical Quantum Gravity 33, 215005 (2016).

[72] R. Emparan, R. Luna, M. Martínez, R. Suzuki, and K. Tanabe, Phases and stability of nonuniform black strings, J. High Energy Phys. 05 (2018) 104.

[73] A. Cisterna and J. Oliva, Exact black strings and p -branes in general relativity, Classical Quantum Gravity 35, 035012 (2018).

[74] P. Kanti, T. Nakas, and N. Pappas, Antigravitating braneworld solutions for a de sitter brane in scalar-tensor gravity, Phys. Rev. D 98, 064025 (2018).

[75] A. Cisterna, C. Corral, and S. del Pino, Static and rotating black strings in dynamical Chern-Simons modified gravity, arXiv:1809.02903.

[76] A. Cisterna, L. Guajardo, and M. Hassaine, Axionic charged black branes with arbitrary scalar nonminimal coupling, arXiv:1901.00514.

[77] M. Bruni, C. Germani, and R. Maartens, Gravitational Collapse on the Brane: A No-Go Theorem, Phys. Rev. Lett. 87, 231302 (2001).

[78] M. Govender and N. Dadhich, Collapsing sphere on the brane radiates, Phys. Lett. B 538, 233 (2002).

[79] T. Tanaka, Classical black hole evaporation in RandallSundrum infinite braneworld, Prog. Theor. Phys. Suppl. 148, 307 (2002).

[80] R. Emparan, A. Fabbri, and N. Kaloper, Quantum black holes as holograms in AdS braneworlds, J. High Energy Phys. 08 (2002) 043.

[81] R. Emparan, J. García-Bellido, and N. Kaloper, Black hole astrophysics in AdS braneworlds, J. High Energy Phys. 01 (2003) 079.

[82] A. Liam Fitzpatrick, L. Randall, and T. Wiseman, On the existence and dynamics of braneworld black holes, J. High Energy Phys. 11 (2006) 033.
[83] R. Gregory, S.F. Ross, and R. Zegers, Classical and quantum gravity of brane black holes, J. High Energy Phys. 09 (2008) 029.

[84] H. Yoshino, On the existence of a static black hole on a brane, J. High Energy Phys. 01 (2009) 068.

[85] D.-C. Dai and D. Stojkovic, Analytic solution for a static black hole in the RSII model, Phys. Lett. B 704, 354 (2011).

[86] S. Bhattacharya and S. R. Kousvos, Constraining the phantom braneworld model from cosmic structure sizes, Phys. Rev. D 96, 104006 (2017).

[87] S. Bhattacharya, S. R. Kousvos, S. Romanopoulos, and T.N. Tomaras, Cosmological screening and the phantom braneworld model, Eur. Phys. J. C 78, 637 (2018).

[88] K. Farakos and P. Pasipoularides, Second Randall-Sundrum brane world scenario with a nonminimally coupled bulk scalar field, Phys. Rev. D 73, 084012 (2006).

[89] C. Bogdanos, A. Dimitriadis, and K. Tamvakis, Brane models with a Ricci-coupled scalar field, Phys. Rev. D 74, 045003 (2006).

[90] K. Farakos and P. Pasipoularides, Gauss-Bonnet gravity, brane world models, and nonminimal coupling, Phys. Rev. D 75, 024018 (2007).

[91] K. Farakos, G. Koutsoumbas, and P. Pasipoularides, Graviton localization and Newton's law for brane models with a nonminimally coupled bulk scalar field, Phys. Rev. D 76, 064025 (2007).

[92] M. Abramowitz and I. A. Stegun, Handbook of Mathematical Functions (Dover Publications, New York, 1972).

[93] P. Binétruy, C. Deffayet, and D. Langlois, Nonconventional cosmology from a brane universe, Nucl. Phys. B565, 269 (2000).

[94] A. Padilla and V. Sivanesan, Boundary terms and junction conditions for generalized scalar-tensor theories, J. High Energy Phys. 08 (2012) 122. 